\title{
Non-perturbative approaches to the quantum Seiberg-Witten curve
}

\author{
Alba Grassi, ${ }^{a}$ Jie $\mathrm{Gu}^{b}$ and Marcos Mariñoo \\ ${ }^{a}$ Simons Center for Geometry and Physics, \\ SUNY, Stony Brook, NY, 1194-3636, U.S.A. \\ ${ }^{b}$ Département de Physique Théorique et Section de Mathématiques, \\ Université de Genève, \\ Genève, $\mathrm{CH}$-1211 Switzerland \\ E-mail: agrassi@scgp.stonybrook.edu, jie.gu@unige.ch, \\ marcos.marino@unige.ch
}

ABSTRACT: We study various non-perturbative approaches to the quantization of the Seiberg-Witten curve of $\mathcal{N}=2, \mathrm{SU}(2)$ super Yang-Mills theory, which is closely related to the modified Mathieu operator. The first approach is based on the quantum WKB periods and their resurgent properties. We show that these properties are encoded in the TBA equations of Gaiotto-Moore-Neitzke determined by the BPS spectrum of the theory, and we relate the Borel-resummed quantum periods to instanton calculus. In addition, we use the TS/ST correspondence to obtain a closed formula for the Fredholm determinant of the modified Mathieu operator. Finally, by using blowup equations, we explain the connection between this operator and the $\tau$ function of Painlevé III.

Keywords: Integrable Hierarchies, Supersymmetric Gauge Theory, Topological Strings, Bethe Ansatz

ARXIV EPRINT: 1908.07065 


\section{Contents}

1 Introduction 1

2 The all-orders WKB method 3

3 Quantum periods from TBA equations $\quad 7$

3.1 Review of the TBA equations of Gaiotto-Moore-Neitzke 8

$\begin{array}{ll}3.2 & \text { TBA equations for the modified Mathieu equation } \\ \end{array}$

$\begin{array}{lll}3.2 .1 & \text { Strong coupling region } & 11\end{array}$

$\begin{array}{ll}3.2 .2 \quad \text { Weak coupling region } & 15\end{array}$

$\begin{array}{ll}\text { 3.3 Solving the TBA equations in the strong coupling region } & 17\end{array}$

4 Quantum periods from instanton calculus $\quad 21$

4.1 Review of instanton calculus 21

4.2 Comparison to Borel resummation 26

5 The Fredholm determinant from topological string theory $\quad 29$

5.1 A closed formula and its derivation 30

5.2 Tests of our formula 32

5.3 Comparison to Zamolodchikov's TBA equation 33

6 On the modified Mathieu operator and Painlevé III $_{3}$

$\begin{array}{lll}7 & \text { Conclusions } & 37\end{array}$

A The four dimensional $\mathrm{SU}(N)$ spectral determinant $\quad 39$

B Zamolodchikov's TBA equation for the modified Mathieu equation 41

\section{Introduction}

In recent years, many interesting and surprising relations have been obtained between quantum mechanical systems, on one hand, and supersymmetric gauge theories and topological strings, on the other hand. One example of such a relation is the gauge/Bethe correspondence of [1], which connects quantum integrable systems to instanton calculus in gauge theory. A second example is the topological string/spectral theory (TS/ST) correspondence, which provides explicit predictions for the spectral determinants of quantum mirror curves [2-4]. Finally, the study of BPS states in supersymmetric gauge theories turns out to be closely related to the WKB method as applied to Seiberg-Witten (SW) curves [5-7]. This relation can be upgraded to include resurgent properties of the quantum 
periods $[8,9]$. All these connections can be used to obtain new results in quantum theory from gauge/string theory. For example, the results of $[1,2]$ lead to new exact quantization conditions for the spectrum of the relevant operators. Conversely, one can use quantum mechanical results to derive new results of string/gauge theories, like for example nonperturbative definitions of topological string partition functions on local Calabi-Yau (CY) manifolds $[2,3,10]$.

Perhaps the simplest quantum-mechanical model where all these methods can be applied is the quantum version of the $\mathrm{SW}$ curve for $\mathcal{N}=2, \mathrm{SU}(2)$ super Yang-Mills (SYM) theory. The corresponding operator is the (modified) Mathieu operator, which is a traditional chapter in the theory of Schrödinger operators. This operator has been also revisited in the context of supersymmetric gauge theory and topological string theory in various works (see e.g. [11-17]), but many important aspects have not been discussed yet. In this paper we use methods from supersymmetric gauge theory and topological string theory to obtain quantum-mechanical properties of the modified Mathieu operator at the non-perturbative level, and we test these properties against first-principles computations. We also discuss the relationships between these different approaches.

The first aspect that we explore is the resurgent structure of the quantum periods, which we review in section 2. Building on [6], Gaiotto considered in [7] the conformal limit of the TBA equations of [5] for an $\mathcal{N}=2$ supersymmetric gauge theory, and he conjectured that the resulting integral equations describe the quantum periods for the corresponding quantum SW curve. In the case of Argyres-Douglas theories, this problem was studied in detail in [8], which pointed out precise connections to the resurgent properties of these periods, and used these properties to derive the conjecture of [7] in the case of general polynomial potentials

In section 3 of this paper we use the conformal limit of the TBA equations to obtain a prediction for these resurgent properties in the case of the modified Mathieu operator. In particular, we obtain the precise structure of the Stokes discontinuities of the quantum periods. We then test these predictions against first-principles calculations in the allorders WKB method, in particular against high order results for the expansion of the quantum periods. We also comment on how to use these TBA equations to compute Borel resummations of the quantum periods.

As pointed out in [11] and explored in many subsequent papers, the NS limit of instanton calculus [1] provides a different resummation of the WKB expansion, in terms of a convergent expansion in the instanton counting parameter. However, this resummation has a very different flavor from the Borel resummation appearing in the theory of resurgence, and it is important to have a precise dictionary between the two types of resummation. We address this issue in section 4.

As we mentioned above, the TS/ST correspondence gives explicit expressions for spectral determinants of operators obtained in the quantization of mirror curves. As pointed out in [18], there is a four-dimensional limit of the correspondence in which the relevant operator is the quantization of the $\mathrm{SW}$ curve for pure $\mathcal{N}=2, \mathrm{SU}(N)$ Yang-Mills theory. This leads to a spectral problem which is different from the one considered in [1] for $N>2$. In the case of the $\mathrm{SU}(2)$ theory considered in this paper, the spectral problems coincide, 
but the TS/ST correspondence gives, in addition to the quantization condition of [1], an explicit expression for the spectral determinant, which we derive in detail in section 5 of this paper. The resummed quantum periods defined by instanton calculus are key ingredients in this expression. We test the resulting formula and in particular we compare our result to the TBA equation describing this spectral determinant which was conjectured by Al. B. Zamolodchikov in [19].

In section 6, based on previous works, we use the vanishing Nakajima-Yoshioka blowup equations to prove that the exact spectrum of the modified Mathieu operator is computed by the zeros of the $\tau$ function of Painlevé $\mathrm{III}_{3}$. Finally, in section 7 we conclude and discuss some open problems.

We have also included two appendices: in the first one we extend the derivation of section 5 to $\mathrm{SU}(N)$ quantum $\mathrm{SW}$ curves, while in the second one we review some of the results of Zamolodchikov's paper [19].

\section{The all-orders WKB method}

Our first approach to the quantum SW curve will be based on the so-called exact WKB method, see for example [20-23]. We will now summarize the basic ingredients of the theory.

The Schrödinger equation for a non-relativistic particle in a potential $V(x)$ and with energy $E$ reads as follows:

$$
-\hbar^{2} \psi^{\prime \prime}(x)+(V(x)-E) \psi(x)=0 .
$$

The standard WKB method produces asymptotic expansions in $\hbar$ for the solutions to this equation. Let us consider the following ansatz for the wavefunction,

$$
\psi(x)=\exp \left(\frac{\mathrm{i}}{\hbar} \int^{x} Y\left(x^{\prime}, E ; \hbar\right) \mathrm{d} x^{\prime}\right) .
$$

The function $Y(x, E ; \hbar)$ satisfies the Riccati equation

$$
Y^{2}-\mathrm{i} \hbar \frac{\mathrm{d} Y}{\mathrm{~d} x}=E-V(x) .
$$

It has the formal power series expansion in powers of $\hbar$

$$
Y(x, E ; \hbar)=\sum_{n=0}^{\infty} p_{n}(x, E) \hbar^{n}
$$

where in particular $p_{0}(x, E)$ is the classical momentum as a function of $x$ and the conserved energy. If one splits $Y$ into the even component and the odd component,

$$
Y=p_{\text {even }}+p_{\text {odd }},
$$

with

$$
p_{\text {even }}(x, E ; \hbar)=\sum_{n=0}^{\infty} p_{2 n}(x, E) \hbar^{2 n}, \quad p_{\text {odd }}(x, E ; \hbar)=\sum_{n=0}^{\infty} p_{2 n+1}(x, E) \hbar^{2 n+1},
$$


one finds that the odd component is in fact a total derivative

$$
p_{\text {odd }}(x, E ; \hbar)=\frac{\mathrm{i} \hbar}{2} \frac{\mathrm{d}}{\mathrm{d} x} \log p_{\text {even }}(x, E ; \hbar) .
$$

By substituting (2.2) into the Schrödinger equation, one finds (see for instance [24])

$$
\begin{aligned}
p_{2 n} & =(-1)^{n} v_{2 n}, \quad n \geq 0 \\
v_{n} & =\frac{1}{2 p_{0}}\left(\partial_{x} v_{n-1}-\sum_{k=1}^{n-1} v_{k} v_{n-k}\right),
\end{aligned}
$$

from which the components $p_{2 n}(x, E)$ can be solved recursively, starting from the known expression of $p_{0}$.

Geometrically, we can regard $p_{\text {even }}(x, E ; \hbar) \mathrm{d} x$ as a meromorphic differential on the curve defined by

$$
y^{2}=2(E-V(x))
$$

We will call it the WKB curve, and we will denote it as $\Sigma_{\mathrm{WKB}}$. This curve depends on a set of moduli which include the energy $E$ and the parameters of the potential $V(x)$. The basic objects in the exact WKB method are the periods of $p_{\text {even }}(x, E ; \hbar) \mathrm{d} x$ along one-cycles of $\Sigma_{\mathrm{WKB}}$, which we will call WKB periods or quantum periods. We will denote them as

$$
\Pi_{\gamma}(\hbar)=\oint_{\gamma} p_{\text {even }}(x, E ; \hbar) \mathrm{d} x, \quad \gamma \in H_{1}\left(\Sigma_{\mathrm{WKB}}\right),
$$

and they are formal power series in even powers of $\hbar$, just like $p_{\text {even }}(x)$,

$$
\Pi_{\gamma}(\hbar)=\sum_{n \geq 0} \Pi_{\gamma}^{(n)} \hbar^{2 n}, \quad \Pi_{\gamma}^{(n)}=\oint_{\gamma} p_{n}(x, E) \mathrm{d} x
$$

Note that the coefficients $\Pi_{\gamma}^{(n)}$ depend on the moduli of the WKB curve. We will call $\Pi_{\gamma}^{(0)}$ the classical periods. The calculation of these coefficients at high order can be quite involved, even for simple quantum systems.

In this paper we are interested in the modified Mathieu Hamiltonian, with the conventions

$$
H(p, x)=p^{2}+V(x), \quad V(x)=2 \Lambda^{2} \cosh x .
$$

Upon quantization, we obtain the operator

$$
\mathrm{H}=\mathrm{p}^{2}+2 \Lambda^{2} \cosh (\mathrm{x}), \quad[\mathrm{x}, \mathrm{p}]=\mathrm{i} \hbar .
$$

We will refer to this as the modified Mathieu operator. It is well-known that the WKB curve of the modified Mathieu Hamiltonian happens to coincide with the SW curve of $\mathcal{N}=2$, $\mathrm{SU}(2)$ Yang-Mills theory, in the conventions appropriate for the relation to integrable systems (see e.g. [25] for a review of SW theory and [26] for its connection to integrable systems). In order to do this, we identify $E$ with the Coulomb modulus $u$ by

$$
E=2 u .
$$


Let us first consider the classical periods of the modified Mathieu equation. Since the WKB curve is a torus, there will be two periods, corresponding to the two cycles of the torus. The $B$ period corresponds to the classical volume of phase space

$$
\Pi_{B}^{(0)}(E)=4 \mathrm{i} \int_{0}^{x_{+}} \mathrm{d} x \sqrt{E-2 \Lambda^{2} \cosh x},
$$

where

$$
x_{+}=\cosh ^{-1} \frac{E}{2 \Lambda^{2}}
$$

is the turning point. This classical period can be evaluated explicitly as

$$
\Pi_{B}^{(0)}(E)=8 \mathrm{i} \sqrt{E+2 \Lambda^{2}}\left[\mathbf{K}\left(\frac{E-2 \Lambda^{2}}{E+2 \Lambda^{2}}\right)-\mathbf{E}\left(\frac{E-2 \Lambda^{2}}{E+2 \Lambda^{2}}\right)\right] .
$$

(We denote the elliptic integrals with boldface letters $\mathbf{K}, \mathbf{E}$, and their argument is the squared modulus $m=k^{2}$ ). There is in addition an $A$ period which corresponds to motion along the imaginary axis. Classically, it is given by,

$$
\Pi_{A}^{(0)}(E)=-2 \mathrm{i} \int_{-\pi \mathrm{i}}^{\pi \mathrm{i}} \mathrm{d} x\left(\sqrt{E-2 \Lambda^{2} \cosh x}\right)=8 \sqrt{E+2 \Lambda^{2}} \mathbf{E}\left(\frac{4 \Lambda^{2}}{2 \Lambda^{2}+E}\right) .
$$

In the simplest case when $E=0$ and $\Lambda=1$, we have

$$
\Pi_{A}^{(0)}(0)=(1+\mathrm{i}) \frac{16 \pi^{3 / 2}}{\Gamma(1 / 4)^{2}}, \quad \Pi_{B}^{(0)}(0)=-\mathrm{i} \frac{16 \pi^{3 / 2}}{\Gamma(1 / 4)^{2}} .
$$

We note that these classical periods are, up to normalization, the famous $a$ and $a_{D}=\partial_{a} F$ periods of SW theory [27], namely

$$
\Pi_{A}^{(0)}(E)=2 \pi a(u), \quad \Pi_{B}^{(0)}(E)=2 \mathrm{i} a_{D}(u) .
$$

We will denote the all-orders WKB quantum periods as

$$
\Pi_{A, B}(E, \hbar)=\sum_{n=0}^{\infty} \hbar^{2 n} \Pi_{A, B}^{(n)}(E) .
$$

In the case of the modified Mathieu equation, the most efficient way to calculate the quantum corrections is the so-called quantum operator approach (see e.g. [13]). It turns out that, for each function $p_{2 n}(x, E)$ appearing in (2.6), one can find a first order differential operator $\mathcal{O}_{n}(E)$ such that

$$
\mathcal{O}_{n}(E) \circ p_{0}(x, E)=p_{2 n}(x, E)
$$

up to a total derivative. Since $\mathcal{O}_{n}(E)$ commutes with integration, one immediately has

$$
\Pi_{A, B}^{(n)}(E)=\mathcal{O}_{n}(E) \circ \Pi_{A, B}^{(0)}(E) .
$$

In this way, we have computed quantum corrections up to order 193. As a simple example, with $\Lambda=1$ we have [13]

$$
\mathcal{O}_{1}(E)=\frac{E}{48\left(4-E^{2}\right)}+\frac{\partial}{24 \partial E} .
$$


Therefore,

$$
\Pi_{A}^{(1)}(E=0)=-\frac{1-\mathrm{i}}{6 \sqrt{2}} \mathbf{K}(-1), \quad \Pi_{B}^{(1)}(E=0)=-\frac{\mathrm{i}}{6 \sqrt{2}} \mathbf{K}(-1) .
$$

We recall that the quantum periods satisfy the so-called quantum Matone relation [14, 17, 28-31]. One of the consequences of this relation is that

$$
\Pi_{A}^{(0)}(E) \Pi_{B}^{(1)}(E)-\Pi_{B}^{(0)}(E) \Pi_{A}^{(1)}(E)=\text { const. },
$$

which we can then evaluate at $E=0$ to be $-2 \pi \mathrm{i} / 3$.

It is well-known that the formal power series appearing in the quantum periods diverge generically as $[24,32]$

$$
\Pi_{\gamma}^{(n)} \approx(2 n) !, \quad n \gg 1 .
$$

Therefore the expressions (2.12) are just formal power series and need to be properly resummed. A natural way of doing so is to perform the Borel resummation. In general, given an asymptotic series of the form

$$
F=\sum_{n=0}^{\infty} f_{n} \hbar^{2 n}, \quad \hbar \in \mathbb{C},
$$

with

$$
f_{n} \sim(2 n) !, \quad n \gg 1
$$

we split $\hbar=\mathrm{e}^{\mathrm{i} \phi}|\hbar|$, and define the Borel resummation to be

$$
s(F)(\hbar)=\frac{1}{|\hbar|} \int_{0}^{\infty} \widehat{F}\left(\mathrm{e}^{\mathrm{i} \phi} \zeta\right) \mathrm{e}^{-\zeta /|\hbar|} \mathrm{d} \zeta,
$$

where $\widehat{F}(\zeta)$ is the Borel transform

$$
\widehat{F}(\zeta)=\sum_{n=0}^{\infty} \frac{f_{n}}{(2 n) !} \zeta^{2 n} .
$$

The analytic properties of $\widehat{F}(\zeta)$ in the $\zeta$-plane, also called the Borel plane, are crucial. If the Borel transform has singularities along the positive real axis, the series $F(\hbar)$ is not Borel summable, as the integral in the Laplace transformation (2.31) is obstructed. We can however deform slightly the integration contour below or above the positive real axis, obtaining in this way the so-called lateral Borel resummations of the formal power series $F(\hbar)$ :

$$
s_{ \pm}(F)(\hbar)=\frac{1}{|\hbar|} \int_{0}^{\mathrm{e}^{\mathrm{i} 0^{ \pm}} \infty} \widehat{F}\left(\mathrm{e}^{\mathrm{i} \phi} \zeta\right) \mathrm{e}^{-\zeta /|\hbar|} \mathrm{d} \zeta .
$$

These lateral resummations are in general different, and their difference is defined as the Stokes discontinuity of $F$ :

$$
\operatorname{disc}(F)(\hbar)=s_{+}(F)(\hbar)-s_{-}(F)(\hbar) .
$$

Stokes discontinuities play a crucial rôle in the theory of resurgence, see e.g. [33]. 


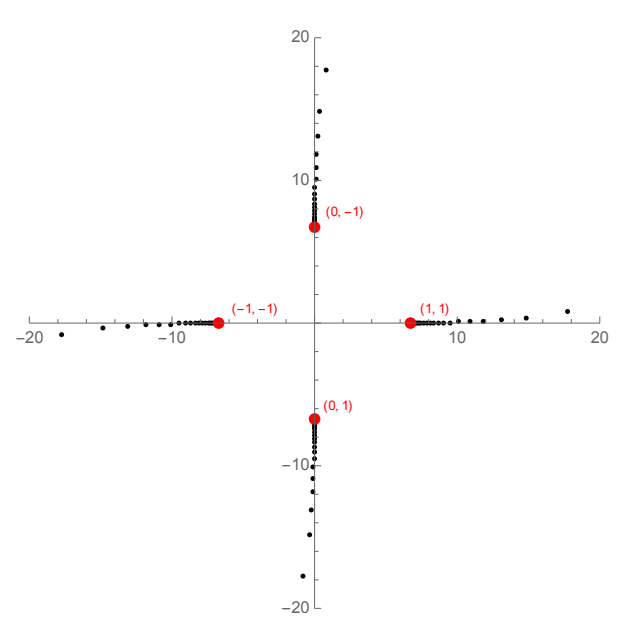

(a) $\Pi_{A}$

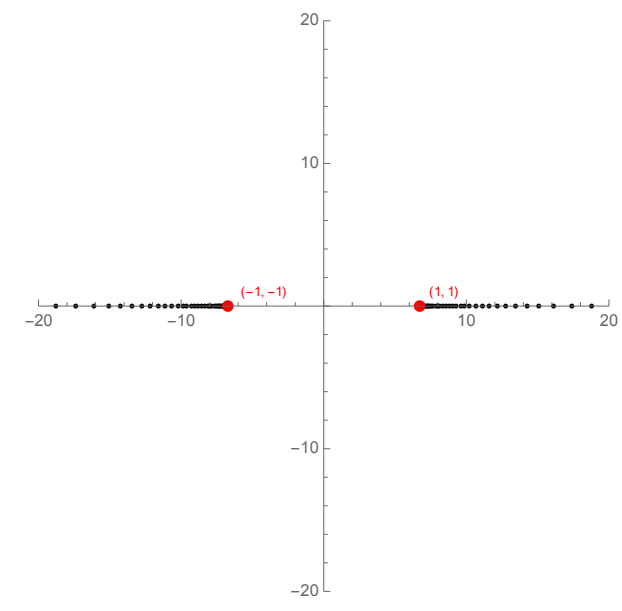

(b) $\Pi_{B}$

Figure 1. Poles of the Borel-Padé transforms, which would accumulate to branch cuts for the Borel transform of the quantum periods $\Pi_{A}(E, \hbar)$ (a) and $\Pi_{B}(E, \hbar)$ (b) at $u=0$ and $\Lambda=1$. The red points are the central charges of the BPS states which contribute to the branch points, and their electromagnetic charges are labelled nearby. See discussion in section 3.2.

Let us look at some examples of the Borel plane of the quantum periods for the modified Mathieu equation. In practice, to calculate the Borel transform, we use standard BorelPadé techniques, i.e. we use a finite number of terms in the formal power series (in this case we have used 193 terms), and in order to extend analytically the resulting function, we use a Padé transform of the Borel transform. In this method, branch cuts of the Borel transform are indicated by a dense accumulation of poles of the Borel-Padé transform along a segment. The first example is when $u=E=0$. We plot the poles of the Borel-Padé transforms of $\Pi_{A}(0, \hbar), \Pi_{B}(0, \hbar)$ in the Borel plane in figures 1 . They indicate the existence of four branch cuts in the case of the $A$ period, and two branch cuts in the case of $B$ period. Since in both cases there are branch cuts along the positive real axis, neither of the two quantum periods are Borel summable.

Next, we consider $u=E / 2=4$. Again we plot the poles of the Borel-Padé transforms of $\Pi_{A}(4, \hbar), \Pi_{B}(4, \hbar)$ in the Borel plane in figures 2. In both cases we observe six branch cuts, and they are in different locations as compared to what we found at $u=0$. In this case, the quantum $A$ period is Borel summable, but the quantum $B$ period is not.

As we can see, in general, the quantum periods are not Borel summable, and their Borel transforms and resummations have a rich structure. Fortunately the connection with SW theory gives very powerful information on this structure, which we will explore in detail in the next section.

\section{Quantum periods from TBA equations}

In this section we study the TBA equations which control the analytic properties of the quantum periods of the modified Mathieu equation. We set $\Lambda=1$ throughout the section. 


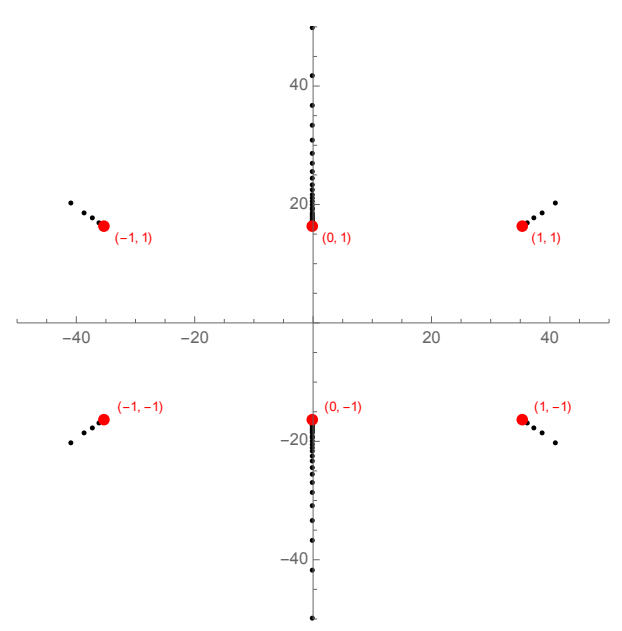

(a) $\Pi_{A}$

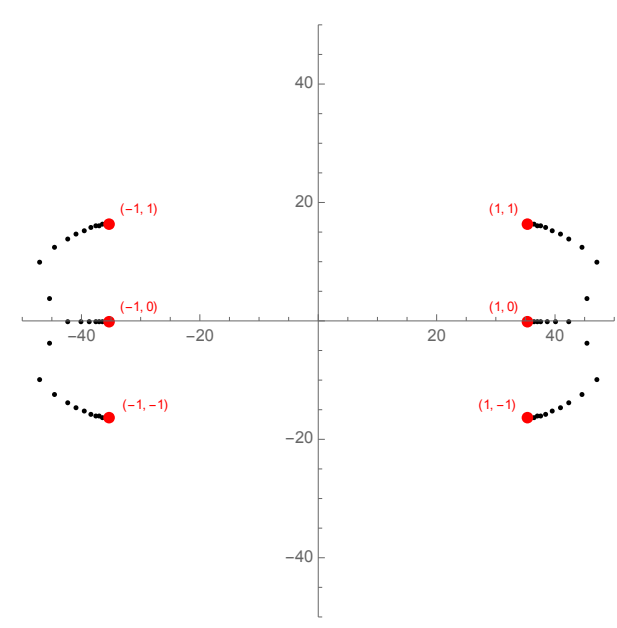

(b) $\Pi_{B}$

Figure 2. Poles of the Borel-Padé transforms, which would accumulate to branch cuts for the Borel transforms of the quantum periods $\Pi_{A}(u, \hbar)$ (a) and $\Pi_{B}(u, \hbar)$ (b) at $u=4$ and $\Lambda=1$. The red points are the central charges of the BPS states which contribute to the branch points, and their electromagnetic charges are labelled nearby. See discussion in section 3.2.

\subsection{Review of the TBA equations of Gaiotto-Moore-Neitzke}

The TBA equations we will obtain are conformal limits [7] of the integral equations proposed by Gaiotto-Moore-Neitzke (GMN) in [5] to describe the hyperKähler metric on the Coulomb branch of $\mathcal{N}=2$ theories compactified on $\mathbb{R}^{3} \times \mathbb{S}_{R}^{1}$, where $R$ is the compactification radius. We will now review some basic aspects of these equations which will be useful in the following. The basic ingredients in these equations are the central charges of the $\mathcal{N}=2$ supersymmetric gauge theory

$$
Z(\boldsymbol{u})=\left(\boldsymbol{a}, \boldsymbol{a}_{D}\right)
$$

where

$$
\boldsymbol{a}_{D}=\frac{\partial F_{0}}{\partial \boldsymbol{a}} .
$$

We define the period associated to a vector $\gamma \in \Gamma$ in the lattice of electromagnetic charges as

$$
Z_{\gamma}=Z(\boldsymbol{u}) \cdot \gamma
$$

This is just a linear combination of $A$ periods and $B$ periods.

To such a central charge we associate a ray

$$
\ell_{\gamma}=\left\{\zeta: \frac{Z_{\gamma}(\boldsymbol{u})}{\zeta} \in \mathbb{R}_{-}\right\}
$$

The semiflat coordinate on the Coulomb branch is given by

$$
\chi_{\gamma}^{\mathrm{sf}}(\zeta)=\exp \left[\pi R \zeta^{-1} Z_{\gamma}+\mathrm{i} \theta_{\gamma}+\pi R \bar{\zeta} Z_{\gamma}\right]
$$


where $R$ is the compactification radius, and

$$
\theta_{\gamma}=\boldsymbol{\theta} \cdot \boldsymbol{\gamma}
$$

is the angular coordinate on the fiber. The semiflat coordinate is the "uncorrected" or "classical" coordinate, and it is corrected by exponentially small effects in the large $R$ limit. These effects are encoded in a non-linear, TBA-like integral equation, which reads as

$$
\chi_{\boldsymbol{\gamma}}(\zeta)=\chi_{\boldsymbol{\gamma}}^{\mathrm{sf}}(\zeta) \exp \left[-\frac{1}{2 \pi \mathrm{i}} \sum_{\gamma^{\prime} \in \Gamma} \Omega\left(\boldsymbol{\gamma}^{\prime} ; \boldsymbol{u}\right)\left\langle\boldsymbol{\gamma}, \boldsymbol{\gamma}^{\prime}\right\rangle \mathcal{I}_{\boldsymbol{\gamma}^{\prime}}(\zeta)\right]
$$

where $\Omega(\gamma ; u)$ is the number of BPS states with electromagnetic charge $\gamma$ at the point $u$ of the Coulomb branch, and

$$
\mathcal{I}_{\gamma}=\int_{\ell_{\gamma}} \frac{\mathrm{d} \zeta^{\prime}}{\zeta^{\prime}} \frac{\zeta^{\prime}+\zeta}{\zeta^{\prime}-\zeta} \log \left(1-\sigma(\gamma) \chi_{\gamma}\left(\zeta^{\prime}\right)\right)
$$

Here, $\sigma(\gamma)$ is the quadratic refinement. It has been argued in [6] that, for BPS hypermultiplets/vectormultiplets, one has, respectively,

$$
\sigma(\gamma)=\mp 1
$$

We have used the normalization of [34], which is more appropriate for our normalization of charges/periods. An important feature of (3.7) is that only those states whose charge $\gamma^{\prime}$ has a non-vanishing Dirac pairing with $\gamma$ contributes to the equation of $\chi_{\gamma}(\zeta)$. The quantities $\chi_{\gamma}(\zeta)$ characterize in a precise way the hyperKähler metric of the moduli space of the $\mathcal{N}=2$ theory compactified on $\mathbb{R}^{3} \times \mathbb{S}_{R}^{1}$, and they can be realized as cluster coordinates on this moduli space [6]. They satisfy the property

$$
\chi_{\gamma+\gamma^{\prime}}(\zeta)=\chi_{\gamma}(\zeta) \chi_{\gamma^{\prime}}(\zeta)
$$

Very often we have both charges $\pm \boldsymbol{\gamma}$ appearing in the sum in the r.h.s. of (3.7). If $\theta_{\gamma}=0$, we have an extra symmetry [7],

$$
\chi_{\gamma}(\zeta)=\chi_{-\gamma}(-\zeta),
$$

and we can combine

$$
\begin{aligned}
\mathcal{C}_{\gamma}=\mathcal{I}_{\gamma}-\mathcal{I}_{-\gamma} & =\int_{\ell_{\gamma}} \frac{\mathrm{d} \zeta^{\prime}}{\zeta^{\prime}} \frac{\zeta^{\prime}+\zeta}{\zeta^{\prime}-\zeta} \log \left(1-\sigma(\gamma) \chi_{\gamma}\left(\zeta^{\prime}\right)\right)-\int_{\ell_{-\gamma}} \frac{\mathrm{d} \zeta^{\prime}}{\zeta^{\prime}} \frac{\zeta^{\prime}+\zeta}{\zeta^{\prime}-\zeta} \log \left(1-\sigma(-\gamma) \chi_{-\gamma}\left(\zeta^{\prime}\right)\right) \\
& =\int_{\ell_{\gamma}} \frac{\mathrm{d} \zeta^{\prime}}{\zeta^{\prime}}\left(\frac{\zeta^{\prime}+\zeta}{\zeta^{\prime}-\zeta}-\frac{\zeta^{\prime}-\zeta}{\zeta^{\prime}+\zeta}\right) \log \left(1-\sigma(\gamma) \chi_{\gamma}\left(\zeta^{\prime}\right)\right) \\
& =4 \zeta \int_{\ell_{\gamma}} \frac{\mathrm{d} \zeta^{\prime}}{\left(\zeta^{\prime}\right)^{2}-\zeta^{2}} \log \left(1-\sigma(\gamma) \chi_{\gamma}\left(\zeta^{\prime}\right)\right)
\end{aligned}
$$

In going from the first to the second line we have changed variables $\zeta^{\prime} \rightarrow-\zeta^{\prime}$, and we used the symmetry (3.11). 


\begin{tabular}{|l|r|}
\hline Resurgence & BPS states \\
\hline WKB curve & SW curve \\
\hline classical limit $\Pi_{\gamma}^{(0)}$ & central charge $Z(\gamma)$ \\
\hline quantum period $\Pi_{\gamma}$ & cluster coordinate $\log \chi_{\gamma}$ \\
\hline Borel singularities & BPS spectrum \\
\hline Stokes discontinuities & KS symplectomorphisms \\
\hline
\end{tabular}

Table 1. Correspondence between the mathematical structures in the resurgent approach to the WKB method, and those in the theory of BPS states.

In order to put the equations in a form similar to the TBA equations, we will perform a change of variables akin to the one made in [5]. If

$$
Z_{\gamma}=\mathrm{e}^{\mathrm{i} \phi^{\prime}}\left|Z_{\gamma}\right|
$$

then we change variables in (3.12) as follows:

$$
\zeta=-\mathrm{e}^{\mathrm{i} \phi-\theta}, \quad \zeta^{\prime}=-\mathrm{e}^{\mathrm{i} \phi^{\prime}-\theta^{\prime}},
$$

and we obtain

$$
\mathcal{C}_{\gamma}=2 \int_{\mathbb{R}} \frac{\log \left(1-\sigma(\gamma) \chi_{\gamma}\left(\theta^{\prime}\right)\right)}{\sinh \left(\theta-\theta^{\prime}+\mathrm{i} \phi^{\prime}-\mathrm{i} \phi\right)} \mathrm{d} \theta^{\prime}
$$

\subsection{TBA equations for the modified Mathieu equation}

Building on $[7,8]$, we expect to have a general correspondence between the mathematical description of BPS states in [5, 6], and the "resurgent" properties of the quantum periods associated to the corresponding SW curve. As noted in [7], this correspondence involves the conformal limit of the TBA equations of [5], which is given by

$$
R \rightarrow 0, \quad \zeta \rightarrow 0, \quad \zeta / R \text { finite }
$$

In this correspondence, the classical limit of the WKB periods $\Pi_{\gamma}^{(0)}$ corresponds to the central charge $Z_{\gamma}$, while the full quantum period is obtained as the logarithm of the Coulomb branch coordinates $\chi_{\gamma}(\zeta)$ (in the conformal limit). The Borel singularities of the Borel transforms $\widehat{\Pi}_{\gamma}$ are closely related to the BPS spectrum of the theory, and the Stokes discontinuities of the quantum periods are closely related to the so-called Kontsevich-Soibelman symplectomorphisms $[5,6,35]$. This correspondence is summarized in table 1 , and it can be used to obtain integral equations of the TBA type governing the quantum periods. We will now apply this correspondence to obtain such equations for the modified Mathieu operator.

Let us then consider the SW theory [27], i.e. pure $\mathcal{N}=2 \mathrm{SYM}$ with gauge group $\mathrm{SU}(2)$. We will denote the charge by

$$
\gamma=\gamma=\left(n_{e}, n_{m}\right)
$$


We will use the conventions of [34] for the symplectic product,

$$
\left\langle\gamma, \gamma^{\prime}\right\rangle=\left\langle\left(n_{e}, n_{m}\right),\left(n_{e}^{\prime}, n_{m}^{\prime}\right)\right\rangle=-n_{e} n_{m}^{\prime}+n_{m} n_{e}^{\prime} .
$$

We will denote

$$
\chi_{e}(\zeta)=\chi_{(1,0)}(\zeta), \quad \chi_{m}(\zeta)=\chi_{(0,1)}(\zeta), \quad \chi_{d}(\zeta)=\chi_{(1,1)}(\zeta)
$$

and because of (3.10) we have

$$
\chi_{d}(\zeta)=\chi_{e}(\zeta) \chi_{m}(\zeta)
$$

We will write TBA equations for $\chi_{e}(\zeta)$ and $\chi_{m}(\zeta)$, as in [34]. We have

$$
\begin{aligned}
& \chi_{e}(\zeta)=\chi_{e}^{\mathrm{sf}}(\zeta) \exp \left[-\frac{1}{2 \pi \mathrm{i}} \sum_{\gamma^{\prime}} c_{e}\left(\gamma^{\prime}\right) \mathcal{I}_{\gamma^{\prime}}(\zeta)\right], \\
& \chi_{m}(\zeta)=\chi_{m}^{\mathrm{sf}}(\zeta) \exp \left[-\frac{1}{2 \pi \mathrm{i}} \sum_{\gamma^{\prime}} c_{m}\left(\gamma^{\prime}\right) \mathcal{I}_{\gamma^{\prime}}(\zeta)\right]
\end{aligned}
$$

where

$$
c_{e}(\gamma)=\Omega(\gamma ; u)\langle(1,0), \gamma\rangle, \quad c_{m}(\gamma)=\Omega(\gamma ; u)\langle(0,1), \gamma\rangle .
$$

In order to write the integral equations, we need to know the structure of the BPS spectrum in SW theory. It is known that there is a curve of marginal stability $\mathcal{C}$ in the Coulomb branch of the SW theory, separating a strong coupling region or chamber $\mathcal{S}$ inside $\mathcal{C}$, from a weak coupling region or chamber $\mathcal{W}$ outside $\mathcal{C}[27,36,37]$. As we move from the strong coupling region to the weak coupling region, the spectrum of BPS states changes drastically by the famous wall-crossing phenomenon. We consider the two chambers in turn.

\subsubsection{Strong coupling region}

We start with the region $\mathcal{W}$ inside the curve of marginal stability. The spectrum consists of one monopole with charge

$$
\gamma_{m}=(0,1)
$$

and one dyon with charge

$$
\gamma_{d}=(1,1)
$$

see $[27,36,37]$ (we follow the conventions in [36]). We also have the corresponding antiparticles, carrying opposite charges. Then, the only nonzero coefficients in (3.21) are

$$
c_{e}\left(\gamma_{m}\right)=c_{e}\left(\gamma_{d}\right)=-1, \quad c_{m}\left(\gamma_{d}\right)=1
$$

Therefore, the equations (3.21) read

$$
\begin{aligned}
\chi_{e}(\zeta) & =\chi_{e}^{\mathrm{sf}}(\zeta) \exp \left[\frac{1}{2 \pi \mathrm{i}}\left(\mathcal{C}_{\gamma_{m}}+\mathcal{C}_{\gamma_{d}}\right)\right], \\
\chi_{m}(\zeta) & =\chi_{m}^{\mathrm{sf}}(\zeta) \exp \left[-\frac{1}{2 \pi \mathrm{i}} \mathcal{C}_{\gamma_{d}}\right],
\end{aligned}
$$


and it is better to write them in terms of $\chi_{d}, \chi_{m}$,

$$
\begin{aligned}
& \chi_{d}(\zeta)=\chi_{d}^{\mathrm{sf}}(\zeta) \exp \left[\frac{1}{2 \pi \mathrm{i}} \mathcal{C}_{\gamma_{m}}\right], \\
& \chi_{m}(\zeta)=\chi_{m}^{\mathrm{sf}}(\zeta) \exp \left[-\frac{1}{2 \pi \mathrm{i}} \mathcal{C}_{\gamma_{d}}\right]
\end{aligned}
$$

We now write the central charges

$$
Z_{d}=\mathrm{e}^{\mathrm{i} \phi_{d}}\left|Z_{d}\right|, \quad Z_{m}=-\mathrm{ie}^{\mathrm{i} \phi_{m}}\left|Z_{m}\right|
$$

These conventions are such that, when $u \in \mathbb{R}$ inside the curve of marginal stability, we have $\phi_{d}=\phi_{m}=0$. Let us define the functions $\epsilon_{d, m}$ and $\widetilde{\epsilon}_{d, m}(\theta)$ as follows (this is similar to the notation used in $[8,38])$ :

$$
\begin{aligned}
& \chi_{d}\left(-\mathrm{e}^{\mathrm{i} \phi_{d}-\theta}\right)=\exp \left(-\epsilon_{d}\left(\theta-\mathrm{i} \phi_{d}\right)\right)=\exp \left(-\widetilde{\epsilon}_{d}(\theta)\right), \\
& \chi_{m}\left(\mathrm{ie}^{\mathrm{i} \phi_{m}-\theta}\right)=\exp \left(-\epsilon_{m}\left(\theta-\mathrm{i} \phi_{m}\right)\right)=\exp \left(-\widetilde{\epsilon}_{m}(\theta)\right) .
\end{aligned}
$$

Then, the conformal limit of the TBA equations reads:

$$
\begin{aligned}
\widetilde{\epsilon}_{d}(\theta) & =\pi\left|Z_{d}\right| \mathrm{e}^{\theta}-2 \int_{\mathbb{R}} \frac{\widetilde{L}_{m}\left(\theta^{\prime}\right)}{\cosh \left(\theta-\theta^{\prime}+\mathrm{i} \phi_{m}-\mathrm{i} \phi_{d}\right)} \frac{\mathrm{d} \theta^{\prime}}{2 \pi}, \\
\widetilde{\epsilon}_{m}(\theta) & =\pi\left|Z_{m}\right| \mathrm{e}^{\theta}-2 \int_{\mathbb{R}} \frac{\widetilde{L}_{d}\left(\theta^{\prime}\right)}{\cosh \left(\theta-\theta^{\prime}+\mathrm{i} \phi_{d}-\mathrm{i} \phi_{m}\right)} \frac{\mathrm{d} \theta^{\prime}}{2 \pi},
\end{aligned}
$$

where we have shifted $\theta \rightarrow \theta-\log R$, and

$$
\widetilde{L}_{m, d}(\theta)=\log \left(1+\mathrm{e}^{-\widetilde{\epsilon}_{m, d}(\theta)}\right) .
$$

We have used here the fact that the BPS spectrum consists of hypermultiplets, therefore $\sigma(\gamma)=-1$.

The equations simplify further when $u$ is real, i.e. $u \in \mathbb{R} \cap \mathcal{W}=[-1,1]$. Then one has $\phi_{d}=\phi_{m}=0$, i.e.

$$
Z_{\gamma_{d}}>0, \quad Z_{\gamma_{m}}=-\mathrm{i}\left|Z_{\gamma_{m}}\right|
$$

and we obtain,

$$
\begin{aligned}
\epsilon_{d}(\theta) & =\pi\left|Z_{d}\right| \mathrm{e}^{\theta}-2 \int_{\mathbb{R}} \frac{L_{m}\left(\theta^{\prime}\right)}{\cosh \left(\theta-\theta^{\prime}\right)} \frac{\mathrm{d} \theta^{\prime}}{2 \pi}, \\
\epsilon_{m}(\theta) & =\pi\left|Z_{m}\right| \mathrm{e}^{\theta}-2 \int_{\mathbb{R}} \frac{L_{d}\left(\theta^{\prime}\right)}{\cosh \left(\theta-\theta^{\prime}\right)} \frac{\mathrm{d} \theta^{\prime}}{2 \pi} .
\end{aligned}
$$

We also note that, before taking the conformal limit, we find the more conventional TBA equations

$$
\begin{aligned}
\epsilon_{d}(\theta) & =\pi r Z_{d} \cosh (\theta)-2 \int_{\mathbb{R}} \frac{L_{m}\left(\theta^{\prime}\right)}{\cosh \left(\theta-\theta^{\prime}\right)} \frac{\mathrm{d} \theta^{\prime}}{2 \pi}, \\
\epsilon_{m}(\theta) & =\pi r\left|Z_{m}\right| \cosh (\theta)-2 \int_{\mathbb{R}} \frac{L_{d}\left(\theta^{\prime}\right)}{\cosh \left(\theta-\theta^{\prime}\right)} \frac{\mathrm{d} \theta^{\prime}}{2 \pi},
\end{aligned}
$$


where

$$
r=2 R .
$$

The definition of $r$ is such that we have the same conventions as in [39].

The TBA equations simplify greatly when $u=0$. In this case, we have that

$$
\left|Z_{d}\right|=\left|Z_{m}\right|=\xi
$$

and that ${ }^{1}$

$$
\epsilon_{d}(\theta)=\epsilon_{m}(\theta)=\epsilon(\theta)
$$

The two TBA equations collapse to one,

$$
\epsilon(\theta)=\pi \xi \mathrm{e}^{\theta}-2 \int_{\mathbb{R}} \frac{L\left(\theta^{\prime}\right)}{\cosh \left(\theta-\theta^{\prime}\right)} \frac{\mathrm{d} \theta^{\prime}}{2 \pi},
$$

which coincides with the integral equation (B.19) associated to the modified Mathieu equation and the Sinh-Gordon model and studied by Zamolodchikov (the factor $\xi$ can be absorbed in a redefinition of the angle $\theta$ ). The equation (3.38) was written down in [7] as governing the quantum periods at $u=0$.

We claim that the functions $\widetilde{\epsilon}_{d, m}(\theta)$ are identified with quantum periods as follows

$$
\begin{aligned}
\widetilde{\epsilon}_{d}\left(x+\mathrm{i} \phi_{d}\right) & =\frac{1}{\hbar} s\left(\Pi_{D}\right)(\hbar), \\
\widetilde{\epsilon}_{m}\left(x+\mathrm{i} \phi_{m}-\frac{\mathrm{i} \pi}{2}\right) & =\frac{1}{\hbar} s\left(\Pi_{B}\right)(\hbar),
\end{aligned}
$$

with

$$
\hbar=\pi^{-1} \mathrm{e}^{-x}, \quad \Pi_{D}=\Pi_{A}+\Pi_{B},
$$

where $\Pi_{D}$ denotes the dyonic quantum period. Then the TBA equations (3.30) are consistent with the leading order contribution by the classical periods in the small $\hbar$ expansion

$$
s\left(\Pi_{D, B}\right)(\hbar)=Z_{d, m}+\mathcal{O}\left(\hbar^{2}\right) .
$$

Furthermore, the TBA equations (3.30) clearly indicate that for some argument angles of $\hbar$ the quantum periods have discontinuities. These discontinuities are determined by the BPS spectrum of SW theory and give the singularity structure of the Borel transform of the quantum periods. These Stokes discontinuities can also be deduced from (3.30). The location of the singularities in the Borel plane, as well as the precise discontinuities, can be checked against the asymptotic series of the quantum periods, by inspecting the Borel plane and by performing lateral Borel resummations, respectively.

\footnotetext{
${ }^{1}$ Anticipating the identification with quantum periods, this equation does not mean that the dyonic and magnetic quamtum periods $\Pi_{D}(u, \hbar), \Pi_{B}(u, \hbar)$ are identical at $u=0$, as $\hbar$ is identified with $\theta$ differently, cf. (3.39).
} 


\begin{tabular}{|cll|}
\hline terms & lateral Borel sum & r.h.s. of $(3.42)$ \\
\hline 181 & 0.17499253901611 & 0.1749925390148815032360 \\
185 & 0.17499253901578 & 0.1749925390148815032482 \\
189 & 0.17499253901545 & 0.1749925390148815032553 \\
193 & $\underline{0.17499253901519}$ & $\underline{0.17499253901488150325} 95$ \\
\hline
\end{tabular}

Table 2. Discontinuity across the ray $\arg (\hbar)=0$ for $\Pi_{B}(u=0, \hbar=1)$ computed by lateral Borel resummation and by using (3.42) with increasing number of terms in the asymptotic series. Underlined are stabilised digits.

For instance, from the TBA equations (3.30), we conclude that $s\left(\Pi_{B}\right)$ are discontinuous across the rays $\arg (\hbar)=\phi_{d}, \phi_{d}+\pi$, with

$$
\begin{aligned}
\operatorname{disc}_{\phi_{d}(+\pi)}\left(\Pi_{B}\right)(\hbar) & =+2 \hbar \log \left(1+\mathrm{e}^{-\frac{1}{\hbar} s\left(\Pi_{D}\right)(\hbar)}\right) \\
& =2 \hbar\left(\mathrm{e}^{-\frac{1}{\hbar} s\left(\Pi_{D}\right)(\hbar)}-\frac{1}{2} \mathrm{e}^{-2 \frac{1}{\hbar} s\left(\Pi_{D}\right)(\hbar)}+\ldots\right) .
\end{aligned}
$$

When $u=0$, we have $\phi_{d}=0$, and the discontinuities are located at $\arg (\hbar)=0, \pi$. The discontinuity across the ray $\arg (\hbar)=0$ can be computed by a lateral Borel resummation of the quantum $B$ period. We check it against the right hand side of (3.42), and find good agreement. See table 2. Similarly, $s\left(\Pi_{D}\right)$ is discontinuous across the rays $\arg (\hbar)=\phi_{m} \pm \frac{\pi}{2}$, and one has

$$
\operatorname{disc}_{\phi_{m} \pm \frac{\pi}{2}}\left(\Pi_{D}\right)(\hbar)=-2 \hbar \log \left(1+\mathrm{e}^{-\frac{1}{\hbar} s\left(\Pi_{B}\right)(\hbar)}\right) .
$$

Numerical checks for this discontinuity formula are completely analogous.

From the discontinuity formula (3.42) we can deduce in the standard way a formula for the large order behavior of $\Pi_{B}^{(n)}$, of the form (see e.g. [40])

$$
\begin{aligned}
\mathrm{i} \Pi_{B}^{(n)} \sim & \frac{2 A^{-2 n+b}}{\pi} \Gamma(2 n+b) \\
& \cdot\left(1-\frac{\mu_{2} A}{2 n+b-1}+\frac{\mu_{3} A^{2}}{(2 n+b-1)(2 n+b-2)}-\frac{\mu_{4} A^{3}}{(2 n+b-1)(2 n+b-2)(2 n+b-3)} \cdots\right) .
\end{aligned}
$$

If we write the dyonic quantum period as

$$
\Pi_{D}=\sum_{n \geq 0} \Pi_{D}^{(n)} \hbar^{2 n}
$$

we can identify

$$
A=\Pi_{D}^{(0)}, \quad b=-1, \quad \mu_{2}=\Pi_{D}^{(1)}, \quad \mu_{3}=\left(\Pi_{D}^{(1)}\right)^{2} / 2, \quad \mu_{4}=\left(\Pi_{D}^{(1)}\right)^{3} / 6+\Pi_{D}^{(2)}, \quad \ldots
$$

These identities are numerically checked at $u=0$ up to all stabilised digits (more than 40) with the help of Richardson transforms.

The large order behavior of $\Pi_{B}^{(n)}$ also indicates that the Borel transform $\widehat{\Pi}_{B}(\zeta)$ has branch points at $\zeta= \pm \Pi_{D}^{(0)}$, which is the central charge of the BPS state (dyon) whose 
electromagnetic charge has non-vanishing Dirac pairing with the charge of the monopole. Similarly, the Borel transform of $\Pi_{A}(u, \hbar)$ should have branch points at the central charges of monopoles and dyons with electromagnetic charges $\pm(0,1), \pm(1,1)$, while the Borel transform of $\Pi_{B}(u, \hbar)$ have branch points only at the central charges of dyons. This explains the Borel plane plots in figure 1, where we also superimpose the central charges of the contributing BPS states as red spots.

\subsubsection{Weak coupling region}

Let us now consider the region outside the curve of marginal stability. The spectrum consists of dyons with charge $\pm \gamma_{n}$, where

$$
\gamma_{n}=(n, 1), \quad n \in \mathbb{Z}
$$

and $W$ boson with charges $\pm \gamma_{e}$, where

$$
\gamma_{e}=(1,0)
$$

From (3.22) we conclude that

$$
c_{e}\left(\gamma_{n}\right)=-1
$$

and

$$
c_{m}\left(\gamma_{n}\right)=n, \quad c_{m}\left(\gamma_{e}\right)=2,
$$

where we used the fact that

$$
\Omega\left(\gamma_{e} ; u\right)=2
$$

in the weak coupling region.

As in [34], we write the equations for $\chi_{e}, \chi_{m}$. We find

$$
\begin{aligned}
& \chi_{m}(\zeta)=\chi_{m}^{\mathrm{sf}}(\zeta) \exp \left[-\frac{1}{\pi \mathrm{i}} \mathcal{C}_{\gamma_{e}}(\zeta)-\frac{1}{2 \pi \mathrm{i}} \sum_{n \in \mathbb{Z}} n \mathcal{C}_{\gamma_{n}}(\zeta)\right] \\
& \chi_{e}(\zeta)=\chi_{e}^{\mathrm{sf}}(\zeta) \exp \left[\frac{1}{2 \pi \mathrm{i}} \sum_{n \in \mathbb{Z}} \mathcal{C}_{\gamma_{n}}(\zeta)\right]
\end{aligned}
$$

In this region we will write

$$
Z_{m}=\mathrm{ie}^{\mathrm{i} \phi_{m}}\left|Z_{m}\right|, \quad Z_{e}=\mathrm{e}^{\mathrm{i} \phi_{e}}\left|Z_{e}\right|, \quad Z_{\ell}=\mathrm{e}^{\mathrm{i} \phi_{\ell}}\left|Z_{\ell}\right|,
$$

where we have denoted $Z_{\ell}=Z_{\gamma_{\ell}}$ the central charge of a dyon. This is chosen in such a way that, if $u$ is real, we have $\phi_{e}=\phi_{m}=0$. We now define

$$
\begin{gathered}
\chi_{e}\left(-\mathrm{e}^{\mathrm{i} \phi_{e}-\theta}\right)=\exp \left(-\epsilon_{e}\left(\theta-\mathrm{i} \phi_{e}\right)\right)=\exp \left(-\widetilde{\epsilon}_{e}(\theta)\right), \\
\chi_{m}\left(-\mathrm{i} \mathrm{e}^{\mathrm{i} \phi_{m}-\theta}\right)=\exp \left(-\epsilon_{m}\left(\theta-\mathrm{i} \phi_{m}\right)\right)=\exp \left(-\widetilde{\epsilon}_{m}(\theta)\right), \\
\chi_{\ell}\left(-\mathrm{e}^{\mathrm{i} \phi_{\ell}-\theta}\right)=\exp \left(-\epsilon_{\ell}\left(\theta-\mathrm{i} \phi_{\ell}\right)\right)=\exp \left(-\widetilde{\epsilon}_{\ell}(\theta)\right),
\end{gathered}
$$


We then obtain the equations,

$$
\begin{gathered}
\widetilde{\epsilon}_{e}(\theta)=\pi\left|Z_{e}\right| \mathrm{e}^{\theta}+\frac{1}{\pi} \int_{\mathbb{R}} \frac{\widetilde{L}_{m}\left(\theta^{\prime}\right)}{\cosh \left(\theta-\theta^{\prime}+\mathrm{i} \phi_{m}-\mathrm{i} \phi_{e}\right)} \mathrm{d} \theta^{\prime}+\frac{1}{\pi \mathrm{i}} \sum_{\ell \neq 0} \int_{\mathbb{R}} \frac{\widetilde{L}_{\ell}\left(\theta^{\prime}\right) \mathrm{d} \theta^{\prime}}{\sinh \left(\theta-\theta^{\prime}+\mathrm{i} \phi_{\ell}-\mathrm{i} \phi_{e}\right)}, \\
\widetilde{\epsilon}_{m}(\theta)=\pi\left|Z_{m}\right| \mathrm{e}^{\theta}-\frac{2}{\pi} \int_{\mathbb{R}} \frac{\widetilde{L}_{e}\left(\theta^{\prime}\right) \mathrm{d} \theta^{\prime}}{\cosh \left(\theta-\theta^{\prime}+\mathrm{i} \phi_{e}-\mathrm{i} \phi_{m}\right)}-\frac{1}{\pi} \sum_{\ell \in \mathbb{Z}} \ell \int_{\mathbb{R}} \frac{\widetilde{L}_{\ell}\left(\theta^{\prime}\right) \mathrm{d} \theta^{\prime}}{\cosh \left(\theta-\theta^{\prime}+\mathrm{i} \phi_{\ell}-\mathrm{i} \phi_{m}\right)}
\end{gathered}
$$

where

$$
\widetilde{L}_{e, m}(\theta)=\log \left(1 \mp \mathrm{e}^{-\widetilde{\epsilon}_{e, m}(\theta)}\right), \quad \widetilde{L}_{\ell}(\theta)=\log \left(1+\mathrm{e}^{-\widetilde{\epsilon}_{\ell}(\theta)}\right) .
$$

Here we have assumed that

$$
\sigma\left(\gamma_{e}\right)=1,
$$

since the $W$ boson is a vector multiplet [6]. In the equation for $\widetilde{\epsilon}_{e}(\theta)$ we have written down explicitly the term corresponding to the dyon with zero electric charge $\gamma_{\ell=0}=\gamma_{m}$, which is the magnetic monopole. We can also deduce the TBA equation for $\widetilde{\epsilon}_{\ell}(\theta)$, by combining the two equations above. We find

$\tilde{\epsilon}_{\ell}(\theta)=\pi\left|Z_{\ell}\right| \mathrm{e}^{\theta}+\frac{2}{\pi \mathrm{i}} \int_{\mathbb{R}} \frac{\widetilde{L}_{e}\left(\theta^{\prime}\right) \mathrm{d} \theta^{\prime}}{\sinh \left(\theta-\theta^{\prime}+\mathrm{i} \phi_{e}-\mathrm{i} \phi_{\ell}\right)}+\frac{1}{\pi \mathrm{i}} \sum_{k \in \mathbb{Z}}(\ell-k) \int_{\mathbb{R}} \frac{\widetilde{L}_{k}\left(\theta^{\prime}\right) \mathrm{d} \theta^{\prime}}{\sinh \left(\theta-\theta^{\prime}+\mathrm{i} \phi_{k}-\mathrm{i} \phi_{\ell}\right)}$.

It is useful to isolate the contribution from the magnetic monopole $k=0$ explicitly in the last term, so that we obtain

$$
\begin{aligned}
\tilde{\epsilon}_{\ell}(\theta)= & \pi\left|Z_{\ell}\right| \mathrm{e}^{\theta}+\frac{2}{\pi \mathrm{i}} \int_{\mathbb{R}} \frac{\widetilde{L}_{e}\left(\theta^{\prime}\right) \mathrm{d} \theta^{\prime}}{\sinh \left(\theta-\theta^{\prime}+\mathrm{i} \phi_{e}-\mathrm{i} \phi_{\ell}\right)}+\frac{1}{\pi \mathrm{i}} \sum_{k \neq 0}(\ell-k) \int_{\mathbb{R}} \frac{\widetilde{L}_{k}\left(\theta^{\prime}\right) \mathrm{d} \theta^{\prime}}{\sinh \left(\theta-\theta^{\prime}+\mathrm{i} \phi_{k}-\mathrm{i} \phi_{\ell}\right)} \\
& -\frac{\ell}{\pi} \int_{\mathbb{R}} \frac{\widetilde{L}_{m}\left(\theta^{\prime}\right) \mathrm{d} \theta^{\prime}}{\cosh \left(\theta-\theta^{\prime}+\mathrm{i} \phi_{m}-\mathrm{i} \phi_{\ell}\right)} .
\end{aligned}
$$

The above equations have some interesting reality properties along the real axis, where $\phi_{e}=\phi_{m}=0$. In that case, since

$$
Z_{\ell}=\ell\left|Z_{e}\right|+\mathrm{i}\left|Z_{m}\right|
$$

one has that

$$
\phi_{\ell}+\phi_{-\ell}=\pi, \quad \ell \in \mathbb{Z} .
$$

It is then easy to see that the conjugation property

$$
\widetilde{\epsilon}_{-\ell}(\theta)=\widetilde{\epsilon}_{\ell}^{*}(\theta)
$$

is compatible with the TBA system. In addition, $\epsilon_{e, m}(\theta)$ are real in this case.

In the weak coupling region, we propose the following identification with quantum periods

$$
\begin{aligned}
\widetilde{\epsilon}_{m}\left(x+\mathrm{i} \phi_{m}+\frac{\mathrm{i} \pi}{2}\right) & =\frac{1}{\hbar} s\left(\Pi_{B}\right)(\hbar), \\
\widetilde{\epsilon}_{e}\left(x+\mathrm{i} \phi_{e}\right) & =\frac{1}{\hbar} s\left(\Pi_{A}\right)(\hbar), \\
\tilde{\epsilon}_{\ell}\left(x+\mathrm{i} \phi_{\ell}\right) & =\frac{1}{\hbar} s\left(\Pi_{\ell}\right)(\hbar),
\end{aligned}
$$


with

$$
\hbar=\pi^{-1} \mathrm{e}^{-x}, \quad \Pi_{\ell}=\ell \Pi_{A}+\Pi_{B} .
$$

The TBA equations (3.55) then imply that the Borel transforms of $\Pi_{A}(\hbar), \Pi_{B}(\hbar)$ have branch points at the central charges of the BPS states whose electromagnetic charges have non-vanishing Dirac pairing with those of the W-boson and monopole, respectively. For $\Pi_{A}(\hbar)$, these are the BPS states with charges $\pm(\ell, \pm 1), \ell=0,1,2, \ldots$; for $\Pi_{B}(\hbar)$, these are the BPS states with charges $\pm(1,0), \pm(\ell, \pm 1), \ell=1,2, \ldots$. This explains the Borel plane plots in figure 2 with $u=4$, well in the weak coupling region. We also superimpose in the plots the central charges of the contributing BPS states as red spots.

In addition, the TBA equations (3.55) also indicate the following discontinuities for the resummed quantum periods $s\left(\Pi_{A}\right)(\hbar), s\left(\Pi_{B}\right)(\hbar)$ in the $\hbar$-plane. The resummed quantum $A$ period $s\left(\Pi_{A}\right)$ is discontinuous

- across the rays $\arg (\hbar)=\phi_{m} \pm \frac{\pi}{2}$ with the discontinuity

$$
\operatorname{disc}_{\phi_{m} \pm \frac{\pi}{2}}\left(\Pi_{A}\right)=-2 \hbar \log \left(1+\mathrm{e}^{-\frac{1}{\hbar} s\left(\Pi_{B}\right)(\hbar)}\right) ;
$$

- across the rays $\arg (\hbar)=\phi_{\ell}(+\pi)$ with the discontinuity

$$
\operatorname{disc}_{\phi_{\ell}(+\pi)}\left(\Pi_{A}\right)=2 \hbar \log \left(1+\mathrm{e}^{-\frac{1}{\hbar} s\left(\Pi_{\ell}\right)(\hbar)}\right), \quad \ell \in \mathbb{Z} .
$$

On the other hand, the quantum $B$ period $s\left(\Pi_{B}\right)$ is discontinuous

- across the rays $\arg (\hbar)=\phi_{e}(+\pi)$ with the discontinuity

$$
\operatorname{disc}_{\phi_{e}(+\pi)}\left(\Pi_{B}\right)=-4 \hbar \log \left(1-\mathrm{e}^{-\frac{1}{\hbar} s\left(\Pi_{A}\right)(\hbar)}\right) ;
$$

- across the rays $\arg (\hbar)=\phi_{\ell}(+\pi)(\ell \neq 0)$ with the discontinuity

$$
\operatorname{disc}_{\phi_{\ell}(+\pi)}\left(\Pi_{B}\right)=-2 \ell \hbar \log \left(1+\mathrm{e}^{-\frac{1}{\hbar} s\left(\Pi_{\ell}\right)(\hbar)}\right), \quad \ell \neq 0 .
$$

To test these formulae, we consider the case of $u=1+4 \mathrm{i}$, where the branch cuts of the Borel transform of quantum $A$ and $B$ periods are well separated, as seen in figure 3 . We compute the discontinuity via lateral Borel resummation for various rays and find good agreement with the r.h.s. of the formulae (3.65)-(3.68), see tables 3, 4 .

Finally, we would like to mention that different TBA-like equations for the quantum periods of the modified Mathieu operator have been proposed in [41] and more recently in [42].

\subsection{Solving the TBA equations in the strong coupling region}

As we have argued, Borel sums of quantum periods are solutions to the TBA equations (3.30), (3.55). In principle the resummed quantum periods can be computed from these TBA equations by using the dictionaries (3.39) and (3.63). In practice, however, 


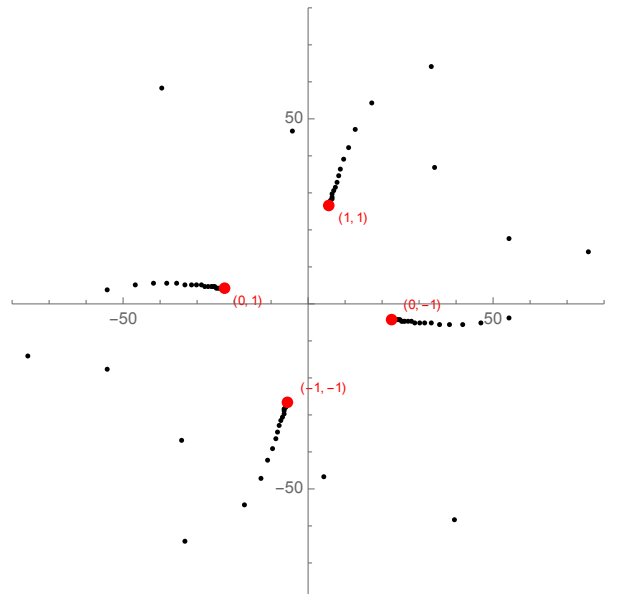

(a) $\Pi_{A}$

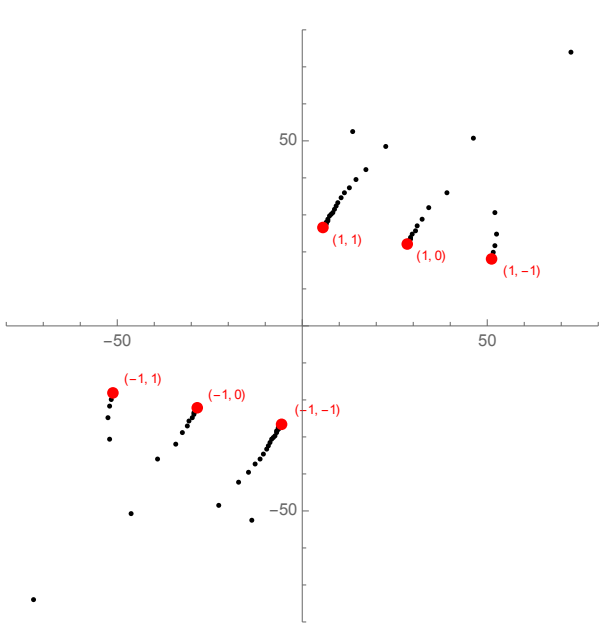

(b) $\Pi_{B}$

Figure 3. Poles of the Borel-Padé transforms, which would accumulate to branch cuts for the Borel transform of the quantum periods $\Pi_{A}(E, \hbar)$ (a) and $\Pi_{B}(E, \hbar)$ (b) at $u=1+4 \mathrm{i}$ and $\Lambda=1$. The red points are the central charges of the BPS states which contribute to the branch points, and their electromagnetic charges are labelled nearby.

\begin{tabular}{|cll|}
\hline BPS state & $(0,-1)$ & $(1,1)$ \\
\hline lateral Borel sum & $1.77819420225 \times 10^{-10}-\mathrm{i} 4.0146843089 \times 10^{-11}$ & $5.37838410 \times 10^{-13}+\mathrm{i} 3.166367886 \times 10^{-12}$ \\
\hline r.h.s. of $(3.65),(3.66)$ & $1.7781942022540 \times 10^{-10}-\mathrm{i} 4.014684308966 \times 10^{-11}$ & $5.3783841037 \times 10^{-13}+\mathrm{i} 3.16636788673 \times 10^{-12}$ \\
\hline
\end{tabular}

Table 3. Discontinuity of $\Pi_{A}(u=1+4 \mathrm{i}, \hbar=1)$ across the rays associated to BPS states $\gamma=$ $(0,-1),(1,1)$, computed by lateral Borel resummation and by using (3.65), (3.66) with up to 193 terms in the asymptotic series. Only stabilised digits are listed.

\begin{tabular}{|cll|}
\hline BPS state & $(1,1)$ & $(1,0)$ \\
\hline lateral Borel sum & $5.37838 \times 10^{-13}+\mathrm{i} 3.166367 \times 10^{-12}$ & $5.979 \times 10^{-16}+\mathrm{i} 4.701 \times 10^{-16}$ \\
\hline r.h.s. of $(3.67),(3.68)$ & $5.3783841 \times 10^{-13}+\mathrm{i} 3.16636788 \times 10^{-12}$ & $5.97982 \times 10^{-16}+\mathrm{i} 4.70119 \times 10^{-16}$ \\
\hline
\end{tabular}

Table 4. Discontinuity of $\Pi_{B}(u=1+4 \mathrm{i}, \hbar=1)$ across the rays associated to BPS states $\gamma=$ $(1,1),(1,0)$, computed by lateral Borel resummation and by using (3.67), (3.68) with up to 193 terms in the asymptotic series. Only stabilised digits are listed.

these equations are difficult to use. First of all, one needs information on the boundary conditions at strong coupling in order to solve the equations. In addition, the standard tools to solve these equations numerically converge very slowly.

Let us first consider the simplest example at $u=0$, where the TBA system collapses to a single equation (3.38), which we reproduce here (we have absorbed the factor $\xi$ in $(3.38)$ in the angle $\theta$ )

$$
\epsilon(\theta)=\pi \mathrm{e}^{\theta}-\int_{\mathbb{R}} \frac{\log \left(1+\mathrm{e}^{-\epsilon\left(\theta^{\prime}\right)}\right)}{\pi \cosh \left(\theta-\theta^{\prime}\right)} \mathrm{d} \theta^{\prime} .
$$

The solution can be identified with the quantum dyon period through the dictionary

$$
\epsilon(\theta)=\frac{1}{\hbar} s\left(\Pi_{D}\right)(u=0, \hbar), \quad \hbar=\Pi_{D}^{(0)} \pi^{-1} \mathrm{e}^{-\theta}=\frac{16 \sqrt{\pi}}{\Gamma\left(\frac{1}{4}\right)^{2}} \mathrm{e}^{-\theta} .
$$


Furthermore, the asymptotic behavior of the solution $\epsilon(\theta)$ as $\theta \rightarrow \infty$ is of the form

$$
\epsilon(\theta) \sim \pi \mathrm{e}^{\theta}+\sum_{n \geq 1} \epsilon^{(n)} \mathrm{e}^{(1-2 n) \theta}
$$

whose coefficients are identified with the quantum corrections to the dyon period

$$
\epsilon^{(n)}=\frac{1}{\pi^{2 n-1}}\left(\Pi_{D}^{(0)}\right)^{2 n-1} \Pi_{D}^{(n)} .
$$

It turns out that the equation (3.69) admits many possible boundary conditions at $\theta \rightarrow-\infty$. This is in stark contrast to the TBA equations for polynomial potentials studied in $[7,8]$, where the equations themselves fix the behavior of the solutions at $\theta \rightarrow-\infty$. One possibility for the boundary conditions at $\theta \rightarrow-\infty$ is the linear behavior (5.28). This type of behavior was considered by Zamolodchikov in [19] but in a slightly different context, as we will discuss in section 5 (see also appendix B). However, it can be seen that this is not well suited for the quantum periods we are studying. ${ }^{2}$ One quick way to see this is that the linear boundary condition with $P \neq 0$ implies $\epsilon^{(1)}<-1 / 3$ (cf. (B.29)), while from the quantum Matone relation (2.27) we find

$$
\epsilon^{(1)}=\frac{1}{\pi} \Pi_{D}^{(0)} \Pi_{D}^{(1)}=\frac{1}{2 \pi \mathrm{i}}\left(\Pi_{A}^{(0)} \Pi_{B}^{(1)}-\Pi_{B}^{(0)} \Pi_{A}^{(1)}\right)=-\frac{1}{3} .
$$

It turns out that the appropriate boundary condition in this case is given by

$$
\epsilon(\theta)=-2 \log \left(-\frac{2 \theta}{\pi}\right)+\cdots, \quad \theta \rightarrow-\infty .
$$

This boundary condition for the TBA equation (3.69) was also studied by Zamolodchikov in [43]. ${ }^{3}$ One can use a small modification of the "dilogarithm trick" of [44] to show that, with the boundary condition (3.74), one has indeed (3.73) (in the context of [43], this calculation gives the central charge $c=1$ for the corresponding sinh-Gordon theory).

To implement numerically the boundary condition (3.74), we borrow a trick from [19]. We define a continuous function

$$
f_{1}(\theta)=-2 \log \left(1+\frac{2}{\pi} \log \left(1+\mathrm{e}^{-\theta}\right)\right)
$$

which has the same boundary behavior as (3.74) and is exponentially suppressed when $\theta \rightarrow+\infty$. We then look for a function $F_{1}(\theta)$ which satisfies

$$
f_{1}(\theta)=\int_{\mathbb{R}} K\left(\theta-\theta^{\prime}\right) F_{1}\left(\theta^{\prime}\right) \mathrm{d} \theta^{\prime}, \quad K(\theta)=\frac{1}{\pi \cosh (\theta)} .
$$

The generic solution to this linear integral equation is

$$
F_{1}(\theta)=\frac{1}{2}\left(f_{1}\left(\theta+\mathrm{i} \frac{\pi}{2}\right)+f_{1}\left(\theta-\mathrm{i} \frac{\pi}{2}\right)\right) .
$$

\footnotetext{
${ }^{2}$ In [7] it was also pointed out that (3.69) admits many boundary conditions at $\theta \rightarrow-\infty$. However, it is claimed there that the correct boundary condition for the quantum period is precisely of the type (B.14), namely, $\epsilon(\theta) \sim \theta / 2+\ldots$ for $\theta \rightarrow-\infty$, which is not quite correct for the reasons explained here.

${ }^{3}$ Alternatively we can justify this boundary condition by using the results in section 5 , see equation (5.40).
} 


\begin{tabular}{|cll|}
\hline & $\hbar=1$ & $\hbar=1 / 2$ \\
\hline TBA & 6.62781 & 13.47880 \\
Borel sum & $6.62781917 \ldots$ & $13.47880936 \ldots$ \\
\hline
\end{tabular}

Table 5. Quantum dyon period at $u=0$.

\begin{tabular}{|cll|}
\hline & $\hbar=1$ & $\hbar=1 / 2$ \\
\hline TBA & 9.16476 & 18.61486 \\
Borel sum & $9.16476545 \ldots$ & $18.61486738 \ldots$ \\
\hline
\end{tabular}

Table 6. Quantum dyon period at $u=1 / 3$.

For our particular $f_{1}(\theta)$, we thus have

$$
F_{1}(\theta)=-\log \left(1+\frac{2}{\pi} \log \left(1+\mathrm{ie}^{-\theta}\right)\right)-\log \left(1+\frac{2}{\pi} \log \left(1-\mathrm{ie}^{-\theta}\right)\right) .
$$

This is a real function for $\theta \in \mathbb{R}$. The TBA equation (3.69) can then be written as

$$
\epsilon(\theta)=\pi \mathrm{e}^{\theta}+f_{1}(\theta)-\int_{\mathbb{R}} \mathrm{d} \theta^{\prime} \frac{1}{\pi \cosh \left(\theta-\theta^{\prime}\right)}\left(\log \left(1+\mathrm{e}^{-\epsilon\left(\theta^{\prime}\right)}\right)+F_{1}\left(\theta^{\prime}\right)\right),
$$

where both boundary conditions at $\pm \infty$ are explicitly spelt out.

The numerical solution to the TBA equation (3.69) converges rather slowly, and we managed to obtain 6 stabilised digits for $\hbar=1$ and 7 stabilised digits for $\hbar=1 / 2$. These results, on the other hand, do agree with the Borel resummation of the quantum dyon period. See table 5 .

Let us now move away from the point $u=0$ but remain in the strong coupling region with $u \in(-1,0) \cup(0,1)$. The TBA system (3.33) has two integral equations coupled to each other. Nevertheless, at $\theta \rightarrow-\infty$ the first terms $\propto \mathrm{e}^{\theta}$ on the r.h.s. of both equations in (3.33) are negligible, and the TBA system also collapses to the single equation (3.69) (with the first term on the r.h.s. suppressed). Therefore both $\epsilon_{d}(\theta), \epsilon_{m}(\theta)$ should have the same boundary condition as (3.74), in other words

$$
\epsilon_{d}(\theta) \sim \epsilon_{m}(\theta) \sim-2 \log \left(-\frac{2 \theta}{\pi}\right)+\ldots, \quad \theta \rightarrow-\infty .
$$

This is corroborated by the fact that the Matone relation (3.73) can be reproduced with this boundary behavior by using again a slight modification of the "dilogarithm trick" [44]. We use again the trick of inserting the pair of $f_{1}(\theta), F_{1}\left(\theta^{\prime}\right)$ functions, and we find that the numerical solution to the TBA system (3.33) has roughly the same speed of convergence as the solution to (3.69) for $u=0$. We tabulate the results for $u=1 / 3$ in tables 6,7 and they also agree with the Borel sum of the quantum periods. Note that the TBA system is solved with $\theta \in \mathbb{R}$, which in light of (3.39) corresponds to real $\hbar$ for the quantum dyon period and to imaginary $\hbar$ for the quantum monopole period. 


\begin{tabular}{|cll|}
\hline & $\hbar=-\mathrm{i}$ & $\hbar=-\mathrm{i} / 2$ \\
\hline TBA & 4.26480 & 8.716486 \\
Borel sum & $4.26480153 \ldots$ & $8.716486917 \ldots$ \\
\hline
\end{tabular}

Table 7. Quantum monopole period at $u=1 / 3$.

\section{Quantum periods from instanton calculus}

Instanton calculus $[1,45]$ leads to a resummation of the quantum periods of the modified Mathieu equation (2.22), as pointed out in [11]. This produces exact functions of $\hbar$ which we will denote by

$$
\Pi_{A, B}^{\mathrm{ex}}(E, \hbar) .
$$

In this section we explain this resummation in detail and we compare it to the Borel resummation obtained in the context of the exact WKB method.

\subsection{Review of instanton calculus}

Let us first review some basic ingredients of instanton calculus in the $4 \mathrm{~d} N=2 \mathrm{SYM}$ with gauge group $G=\mathrm{SU}(N)$ [1, 45-48].

We denote a partition (or Young tableaux) by

$$
Y=\left(y_{1}, y_{2}, \cdots\right)
$$

its transposed by

$$
Y^{t}=\left(y_{1}^{t}, y_{2}^{t}, \cdots\right)
$$

and a vector of Young tableaux as

$$
\boldsymbol{Y}=\left(Y_{1}, \cdots, Y_{N}\right)
$$

It is useful to define

$$
h_{Y}(s)=y_{i}-j, \quad v_{Y}(s)=y_{j}^{t}-i,
$$

where $s=(i, j)$ is a box (not necessarily in the partition $Y$ ). We will also use

$$
\ell(\boldsymbol{Y})=\sum_{I=1}^{N} \ell\left(Y_{I}\right)
$$

whith

$$
\ell(Y)=\sum_{i} y_{i}
$$

The four dimensional $\mathrm{SU}(N)$ Nekrasov partition function is $[45,47]$

$$
Z\left(\boldsymbol{a} ; \epsilon_{1}, \epsilon_{2}\right)=\sum_{\boldsymbol{Y}}\left((-1)^{N} \Lambda^{2 N}\right)^{\ell(\boldsymbol{Y})} \mathcal{Z}_{\boldsymbol{Y}}
$$


where

$$
\begin{aligned}
\mathcal{Z}_{\boldsymbol{Y}}=\prod_{I, J=1}^{N} \prod_{s \in Y_{I}} \frac{1}{\alpha_{I}-\alpha_{J}-\epsilon_{1} v_{Y_{J}}(s)+\epsilon_{2}\left(h_{Y_{I}}(s)+1\right)} \\
\times \prod_{s \in Y_{J}} \frac{1}{\alpha_{I}-\alpha_{J}+\epsilon_{1}\left(v_{Y_{I}}(s)+1\right)-\epsilon_{2} h_{Y_{J}}(s)},
\end{aligned}
$$

with

$$
\sum_{i=1}^{N} \alpha_{i}=0
$$

and

$$
a_{i}=\alpha_{i}-\alpha_{i+1}, \quad i=1, \cdots, N-1 .
$$

The four dimensional SU $(N)$ Nekrasov-Shatashvili (NS) free energy is then defined by [1]

$$
F_{\mathrm{NS}}^{\mathrm{inst}}(\boldsymbol{a}, \hbar)=\mathrm{i} \hbar \lim _{\epsilon_{2} \rightarrow 0} \epsilon_{2} \log Z\left(\boldsymbol{a} ; \mathrm{i} \hbar, \epsilon_{2}\right) .
$$

An important property of this free energy is that it is given as a power series in $\Lambda$ which is expected to have a non-vanishing radius of convergence in a certain range of values of $a$ and $\hbar$ around the semiclassical region $|\Lambda / a| \ll 1$. $^{4}$

In order to make contact with the (modified) Mathieu equation, the relevant gauge theory is $\mathcal{N}=2, \mathrm{SU}(2) \mathrm{SYM}$ theory, hence we have to consider (4.12) with $N=2$. In this case the first few terms read

$$
F_{\mathrm{NS}}^{\mathrm{inst}}(a, \hbar)=-\frac{2 \Lambda^{4}}{a^{2}+\hbar^{2}}+\frac{\Lambda^{8}\left(7 \hbar^{2}-5 a^{2}\right)}{\left(a^{2}+\hbar^{2}\right)^{3}\left(a^{2}+4 \hbar^{2}\right)}+\mathcal{O}\left(\Lambda^{16}\right) .
$$

Once the NS free energy is known, the quantum $A$ period $a(u, \hbar)$ can be obtained by inverting the quantum Matone relation [29, 49-54]

$$
u=\frac{a^{2}}{8}-\frac{\Lambda}{8} \partial_{\Lambda} F_{\mathrm{NS}}^{\mathrm{inst}}(a, \hbar, \Lambda) .
$$

This leads to a series expansion for $a(u, \hbar)$ in powers of $\Lambda^{4}$ which is expected to converge in an appropriate range of the parameters $\Lambda, E, \hbar$ around the semiclassical region $\left|\Lambda^{2} / E\right| \ll 1$. The quantum $B$ period $a_{D}(u, \hbar)$ is then obtained from the quantum special geometry relation

$$
a_{D}(a, \hbar)=\partial_{a} F_{\mathrm{NS}}(a, \hbar, \Lambda)=2 \gamma(a, \hbar, \Lambda)+\partial_{a} F_{\mathrm{NS}}^{\mathrm{inst}}(a, \hbar, \Lambda)
$$

where $[55]$

$$
\gamma(a, \hbar)=\frac{a}{2} \log \left(\frac{\hbar^{2}}{\Lambda^{2}}\right)-\frac{\pi \hbar}{4}-\frac{\mathrm{i} \hbar}{2}\left(\log \Gamma\left(1+\frac{\mathrm{i} a}{\hbar}\right)-\log \Gamma\left(1-\frac{\mathrm{i} a}{\hbar}\right)\right),
$$

and we replace $a$ by $a(u, \hbar)$.

\footnotetext{
${ }^{4}$ Recall that for $\hbar=0$, in the electric frame both $a(u)$ and $a_{D}(u)$ are convergent series of $\Lambda^{2} / u$ up to the monopole and dyon points. The prepotential, which is related to $a, a_{D}$ by the special geometry relation, is thus also convergent series of $\Lambda / a$ up to these points. The NS free energy is its smooth deformation which tends to enlarge the domain of convergence.
} 
We also note that it is possible to express the NS free energy via a TBA system $[1,56]$ (different from the one discussed in section 3.2). This TBA system however has a range of validity/convergence which is smaller than the one of the instanton calculus. For instance, the TBA breaks down if $\operatorname{Re}(a) \neq 0$, while the instanton counting expression for $F_{\mathrm{NS}}^{\text {inst }}(4.12)$ is still well defined.

It was found in [11] that by expanding (4.13), (4.14) at small values of $\hbar$, it is possible to recover the WKB periods (2.22). More precisely, at order $\hbar^{2 n}$, one finds an expansion in $\Lambda$ which agrees with the expansion of $\Pi^{(n)}(E)$ at large $E$ :

$$
\begin{aligned}
& 2 \pi a(u, \hbar) \longrightarrow \sum_{n=0}^{\infty} \hbar^{2 n} \Pi_{A}^{(n)}(E=2 u), \\
& 2 \mathrm{i} a_{D}(a(u, \hbar), \hbar)=\left.2 \mathrm{i} \partial_{a}\left(F_{\mathrm{NS}}(a, \hbar)\right)\right|_{a=a(u, \hbar)} \longrightarrow \sum_{n=0}^{\infty} \hbar^{2 n} \Pi_{B}^{(n)}(E=2 u) .
\end{aligned}
$$

Therefore, the Bethe/gauge correspondence provides an analytic way to resum the WKB periods period into well defined functions which are exact in $\hbar$. We will denote these functions by $\Pi_{A, B}^{\mathrm{ex}}(E, \hbar)$, and we will refer to them as "exact" quantum periods. In terms of the quantities that we have introduced, they are given by

$$
\begin{aligned}
& \Pi_{A}^{\mathrm{ex}}(E, \hbar)=2 \pi a(E, \hbar), \\
& \Pi_{B}^{\mathrm{ex}}(E, \hbar)=2 \mathrm{i} \partial_{a} F_{\mathrm{NS}}(a(E, \hbar), \hbar),
\end{aligned}
$$

where we use the notation $a(E, \hbar):=a(u=E / 2, \hbar)$.

It turns out that one can find the series expansion for $a(u, \hbar)$ by using elementary methods. To do this, we use the WKB method, but we solve the Riccati equation (2.3) perturbatively in $\Lambda$, i.e. we solve

$$
Y^{2}(x)-\mathrm{i} \hbar \frac{\mathrm{d} Y(x)}{\mathrm{d} x}=E-2 \Lambda^{2} \cosh (x)
$$

with an ansatz

$$
Y(x)=\sum_{n \geq 0} Y_{n}(x, \hbar, E) \Lambda^{2 n}
$$

Clearly, we should set

$$
Y_{0}(x, \hbar, E)=\sqrt{E}
$$

The equation for $Y_{1}(x, \hbar, E)$ is

$$
2 Y_{0} Y_{1}-\mathrm{i} \hbar \frac{\mathrm{d} Y_{1}}{\mathrm{~d} x}=-2 \cosh (x)
$$

The general solution to this equation is of the form

$$
Y_{1}(x, \hbar, E)=-\frac{4 \sqrt{E} \cosh (x)+2 \mathrm{i} \hbar \sinh (x)}{4 E+\hbar^{2}}+c \mathrm{e}^{-\frac{2 \mathrm{i} \sqrt{E} x}{\hbar}} .
$$

We note that the term involving the unknown coefficient leads to a non-perturbative effect in $\hbar$. We will set it to zero to recover the perturbative series. The general term $Y_{n}$ satisfies

$$
2 \sqrt{E} Y_{n}(x, \hbar, E)-\mathrm{i} \hbar \frac{\mathrm{d} Y_{n}}{\mathrm{~d} x}+\sum_{k=1}^{n-1} Y_{k}(x, \hbar, E) Y_{n-k}(x, \hbar, E)=0 .
$$


This can be integrated order by order, setting to zero non-perturbative terms. We find in this way,

$$
Y_{2}(x, \hbar, E)=-\frac{4 E^{2}+5 E \hbar^{2}+\mathrm{i} \sqrt{E} \hbar\left(8 E-\hbar^{2}\right) \sinh (2 x)+E\left(4 E-5 \hbar^{2}\right) \cosh (2 x)+\hbar^{4}}{\sqrt{E}\left(E+\hbar^{2}\right)\left(4 E+\hbar^{2}\right)^{2}} .
$$

The functions $Y_{n}(x, \hbar, E)$ are complicated, but their integrals are slightly simpler. As it follows from (2.19), we have to calculate

$$
\mathcal{I}_{n}(E, \hbar)=\frac{1}{2 \pi} \int_{-\pi}^{\pi} Y_{n}(\mathrm{i} x, \hbar, E) \mathrm{d} x
$$

As expected, only even terms contribute. We find, for example,

$$
\begin{aligned}
& \mathcal{I}_{2}(E, \hbar)=-\frac{1}{4 E^{3 / 2}+\sqrt{E} \hbar^{2}}, \\
& \mathcal{I}_{4}(E, \hbar)=-\frac{60 E^{2}+35 E \hbar^{2}+2 \hbar^{4}}{4 E^{3 / 2}\left(E+\hbar^{2}\right)\left(4 E+\hbar^{2}\right)^{3}}, \\
& \mathcal{I}_{6}(E, \hbar)=-\frac{6720 E^{5}+18480 E^{4} \hbar^{2}+15260 E^{3} \hbar^{4}+4705 E^{2} \hbar^{6}+413 E \hbar^{8}+18 \hbar^{10}}{4 E^{5 / 2}\left(E+\hbar^{2}\right)^{2}\left(4 E+\hbar^{2}\right)^{5}\left(4 E+9 \hbar^{2}\right)},
\end{aligned}
$$

and so on. Then, one finds

$$
a(E, \hbar)=2\left(\sqrt{E}+\sum_{m=1}^{\infty} \mathcal{I}_{2 m}(E, \hbar) \Lambda^{4 m}\right) .
$$

When $\hbar=0$, we recover the standard SW period (2.19):

$$
2 \pi a(E, 0)=4 \pi \sqrt{2+E}{ }_{2} F_{1}\left(-\frac{1}{2}, \frac{1}{2}, 1 ; \frac{4}{2+E}\right)=8 \sqrt{2 \Lambda^{2}+E} \mathbf{E}\left(\frac{4 \Lambda^{2}}{2 \Lambda^{2}+E}\right) .
$$

At finite $\hbar, \Lambda$ we also find perfect agreement between (4.28) and the standard result of instanton calculus.

We note that the integrals above can be calculated as residues, since

$$
\mathcal{I}_{n}(E, \hbar)=\oint_{|X|=1} Y_{n}(X, \hbar, E) \frac{\mathrm{d} X}{X}=\operatorname{Res}_{X=0} \frac{Y_{n}(X, \hbar, E)}{X},
$$

where

$$
X=\mathrm{e}^{x} .
$$

In fact, it is more convenient to solve the differential equation directly in the $X$ variable, since everything is algebraic, i.e. it is better to solve

$$
2 \sqrt{E} Y_{n}(X, \hbar, E)-\mathrm{i} \hbar X \frac{\mathrm{d} Y_{n}}{\mathrm{~d} X}(X, \hbar, E)+\sum_{k=1}^{n-1} Y_{k}(X, \hbar, E) Y_{n-k}(X, \hbar, E)=0 .
$$

It turns out that the function $Y(\mathrm{i} x, \hbar, E)$ can be calculated exactly in terms of Mathieu functions. To see this, we note that $\mathcal{Y}(x, \hbar, E)=Y(\mathrm{i} x, \hbar, E)$ satisfies the Riccati equation

$$
\mathcal{Y}^{2}(x, \hbar, E)-\hbar \frac{\mathrm{d} \mathcal{Y}}{\mathrm{d} x}(x, \hbar, E)=E-2 \Lambda^{2} \cos (x),
$$


which is the Mathieu equation with imaginary Planck constant. The solution to this equation is

$$
\mathcal{Y}(x, \hbar, E)=-\hbar \frac{\mathrm{d}}{\mathrm{d} x} \log \left\{S\left(-\frac{4 E}{\hbar^{2}},-\frac{4 \Lambda^{2}}{\hbar^{2}}, \frac{x}{2}\right)+c C\left(-\frac{4 E}{\hbar^{2}},-\frac{4 \Lambda^{2}}{\hbar^{2}}, \frac{x}{2}\right)\right\},
$$

where $c$ is an integration constant and $S(\alpha, q, x), C(\alpha, q, x)$ are the odd (even) Mathieu functions, respectively. Since we want

$$
\mathcal{Y}(x, \hbar, E) \approx \sqrt{E}
$$

as $\Lambda \rightarrow 0$, we find that this leads to

$$
c= \pm \mathrm{i}
$$

(where the sign depends on the branch cut of the square root). We eventually find

$$
\Pi_{A}^{\mathrm{ex}}(E, \hbar)=2 \int_{-\pi}^{\pi} \mathcal{Y}(x) \mathrm{d} x=-2 \hbar \log \frac{S\left(-\frac{4 E}{\hbar^{2}},-\frac{4 \Lambda^{2}}{\hbar^{2}}, \frac{\pi}{2}\right)+c C\left(-\frac{4 E}{\hbar^{2}},-\frac{4 \Lambda^{2}}{\hbar^{2}}, \frac{\pi}{2}\right)}{-S\left(-\frac{4 E}{\hbar^{2}},-\frac{4 \Lambda^{2}}{\hbar^{2}}, \frac{\pi}{2}\right)+c C\left(-\frac{4 E}{\hbar^{2}},-\frac{4 \Lambda^{2}}{\hbar^{2}}, \frac{\pi}{2}\right)} .
$$

Note that

$$
\Pi_{A}^{\mathrm{ex}}(E, \hbar)=2 \pi \mathrm{i} \hbar \nu\left(-\frac{4 E}{\hbar^{2}},-\frac{4 \Lambda^{2}}{\hbar^{2}}\right),
$$

where $\nu$ is the characteristic exponent of the Mathieu equation (this relation has been noted in the context of the Mathieu equation in e.g. $[12,15])$. The advantage of the expressions (4.37), (4.38) is that they make sense for values of $E$ for which the $\Lambda$ expansion (4.28) does not converge, so they extend (4.28) to a larger domain.

Let us consider some numerical examples. When $E=100, \hbar=3 \pi$ and $\Lambda=1$, we can evaluate the series (4.28) by truncating it up to order $\Lambda^{28}$, and we find:

$$
\frac{1}{4 \pi} \Pi_{A}^{\mathrm{ex}}(100,3 \pi)=-9.9997954179096157891757 \ldots
$$

This is precisely what is also obtained from (4.37) and (4.38). At the same time, by using (4.37) we can go all the way to $E=0$, where (4.28) cannot be used. We find, for example,

$$
\frac{1}{4 \pi} \Pi_{A}^{\mathrm{ex}}(0,3 \pi)=0.1501122164563802133431995 \ldots \mathrm{i} .
$$

This procedure for evaluating the value of $\Pi_{A}^{\mathrm{ex}}$ at $E=0$ seems to be well-defined for sufficiently large $\hbar \Lambda^{-2}$ (e.g. $\hbar \Lambda^{-2} \geq 1$ works).

We conclude that the "exact" quantum $A$ period can be computed either by the expression given by instanton calculus (or equivalently, by the closely related series (4.28)), or by the expression (4.38) involving the Mathieu characteristic exponent (4.38). When these two expressions are both well-defined, they agree, but (4.38) has a larger range of validity. In the case of the quantum $B$ period, it might be possible to obtain an alternative expression to the one in (4.18), in terms of infinite Hill determinants, by using results in [57]. 


\begin{tabular}{|cc|}
\hline terms included & Borel sum \\
\hline 190 & 35.40661948105291481767982565157 \\
191 & $\underline{35.40661948105291481767982565207}$ \\
\hline gauge theory & 35.40661948105291481767982564492 \\
\hline
\end{tabular}

Table 8. Borel sum of quantum $A$ period at $u=4$ and $\hbar=1$. Underlined are stabilised digits.

\subsection{Comparison to Borel resummation}

We now have two different approaches to the calculation of (resummed) quantum periods: on the one hand, we have the Borel resummation of the all-orders WKB expansion in $\hbar^{2}$, which is also calculated by the TBA equations of section 3. On the other hand, instanton calculus gives a different resummation, based on a convergent expansion in $\Lambda$, as a function of $\hbar$. An obvious question is: what is the precise relation between these two resummations? Since both lead to the same asymptotic expansion in powers of $\hbar$, we expect that they will differ in non-perturbative effects. In this section we will address this issue. Results along these lines have been previously obtained in $[15,16]$. For simplicity, we will restrict ourselves to the case in which $\hbar>0$ and $u$ is real.

Let us first consider the weak coupling region $\mathcal{W}$. Here, the all-orders WKB quantum $A$ period is Borel summable for $\hbar$ real, and we find that its Borel sum agrees well with $2 \pi a(u, \hbar)$ obtained by inverting (4.14) or with the solution (4.37) to the Riccati equation, i.e.

$$
s\left(\Pi_{A}\right)(E, \hbar)=\Pi_{A}^{\mathrm{ex}}(E, \hbar), \quad|u|>1, \quad \hbar>0 .
$$

We illustrate this in table 8 where we compare the Borel sum of the WKB quantum $A$ period at $u=4$ and $\hbar=1$, with increasing number of corrections, to the result of instanton calculus. They agree with almost all the stabilised digits (27 of them).

The quantum $B$ period, on the other hand, is not Borel summable along the real axis in the weak coupling region. Nevertheless, we can make the following observation. The "exact" quantum $B$ period is given by

$$
2 \mathrm{i} a_{D}(u, \hbar)=4 \mathrm{i} \gamma(a, \hbar)+2 \mathrm{i} \partial_{a} F_{\mathrm{NS}}^{\mathrm{inst}}(a, \hbar)
$$

where $\gamma$ is defined in (4.16) and has the following asymptotic expansion for large $a / \hbar$ :

$$
\gamma(a, \hbar) \sim a\left(\log \left(\frac{a}{\Lambda}\right)-1\right)+a \sum_{n \geq 1} \frac{(-1)^{n} B_{2 n}}{2 n(2 n-1)}\left(\frac{\hbar}{a}\right)^{2 n} .
$$

The series in $\hbar / a$ in the r.h.s. is not Borel summable along the positive real axis. More precisely, let us consider the following formal power series:

$$
\varphi(z)=\sum_{n \geq 0} c_{n} z^{2 n}, \quad c_{n-1}=\frac{(-1)^{n} B_{2 n}}{2 n(2 n-1)} .
$$

A little numerical experimentation shows that the lateral resummations of this series along the positive real axis are given by

$$
s_{ \pm}(\varphi)(z)=f(z) \pm \frac{\mathrm{i}}{2 z} \log \left(1-\mathrm{e}^{-2 \pi / z}\right),
$$




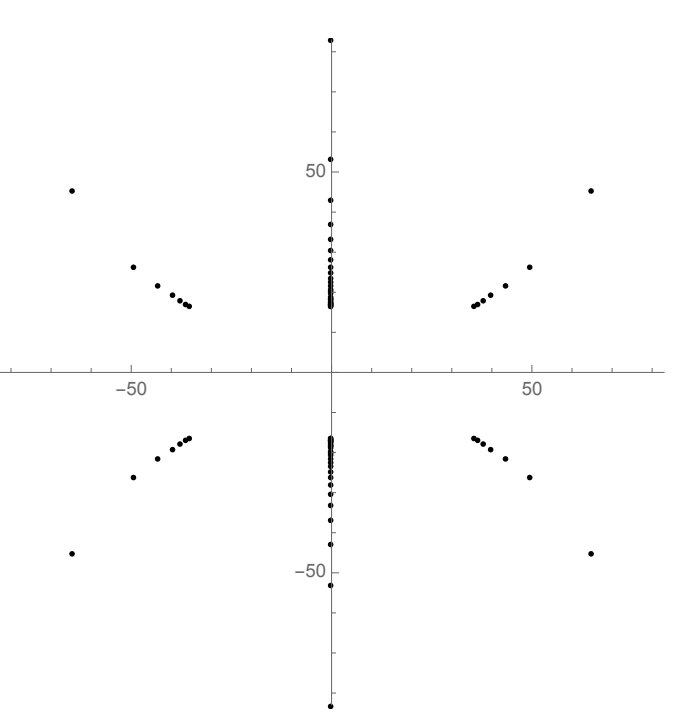

Figure 4. Poles of the Borel-Padé transform of the "reduced" series $\prod_{B}^{\mathrm{red}}$ at $u=4$.

where

$$
f(z)=\frac{1}{z^{2}}(\log (z)+1)-\frac{\pi}{4 z}-\frac{\mathrm{i}}{2 z} \log \frac{\Gamma(1+\mathrm{i} / z)}{\Gamma(1-\mathrm{i} / z)} .
$$

(A similar series has been considered in [55]).

The above analysis suggests that the non-Borel summability of the sequence $\Pi_{B}^{(n)}$ along the positive real axis in the weak coupling region is due to the asymptotic series appearing in $\gamma(a, \hbar)$. In view of (4.45), this leads to the right discontinuity across the positive real axis:

$$
-4 \hbar \log \left(1-\mathrm{e}^{-4 \pi a / \hbar}\right)
$$

This suggests that the "reduced" formal power series

$$
\Pi_{B}^{\mathrm{red}}(E, \hbar):=\Pi_{B}(E, \hbar)-4 \mathrm{i} \Pi_{A}(E, \hbar) \sum_{n \geq 1} \frac{(-1)^{n} B_{2 n}}{2 n(2 n-1)}\left(\frac{\hbar}{2 \Pi_{A}(E, \hbar)}\right)^{2 n},
$$

where we subtract the non-Borel summable series in the function $\gamma(a, \hbar)$, is actually Borel summable along the positive real axis. We verified numerically that this indeed is true, as can be seen from the Borel plane plot at $u=4$ given in figure 4 . In fact the Borel sum of $\Pi_{B}^{\mathrm{red}}(E, \hbar)$ agrees with the gauge theory calculation in which the contribution of $\gamma(a, \hbar)$ has been removed; in other words,

$$
s\left(\Pi_{B}^{\mathrm{red}}\right)(E, \hbar)=2 \mathrm{i} a_{D}^{\mathrm{red}}(u, \hbar):=4 \mathrm{i} a\left(\log \left(\frac{a}{\Lambda}\right)-1\right)+2 \mathrm{i} \frac{\partial F_{\mathrm{NS}}^{\mathrm{ins}}(a, \hbar)}{\partial a} .
$$

We illustrate this in table 9 , where both the Borel sum of $\Pi_{B}^{\mathrm{red}}(E, \hbar)$ and $2 \mathrm{i} a_{D}^{\mathrm{red}}(u, \hbar)$ are evaluated at $u=4$ and $\hbar=1$. We find that all stabilised digits are in agreement (26 of them)..$^{5}$

\footnotetext{
${ }^{5}$ We also notice that the exact $B$ period $\Pi_{B}^{\text {ex }}(E, \hbar)$ with $E>2$ agrees with the average of lateral Borel resummations of the quantum $B$ period $\Pi_{B}(E, \hbar)$.
} 


\begin{tabular}{|ccl|}
\hline & terms \\
\hline$\Pi_{B}^{\mathrm{red}}$ & 159 & 16.474810551500808917635392219 \\
& 161 & $\underline{16.474810551500808917635392368}$ \\
\hline $2 \mathrm{i} a_{D}^{\mathrm{red}}$ & 14 & 16.47481055150080891763539232909 \\
& 15 & $\underline{16.47481055150080891763539232920}$ \\
\hline
\end{tabular}

Table 9. Comparison between the Borel sum of the "reduced" quantum $B$ period $\Pi_{B}^{\text {red }}$ at $u=4$ and $\hbar=1$ (with increasing number of terms), and the exact, "reduced" period $2 \mathrm{i} a_{D}^{\text {red }}$ defined in (4.49) (with increasing number of instanton corrections). Underlined are stabilised digits.

\begin{tabular}{|cl|}
\hline terms & first line of r.h.s. of $(4.50)$ \\
\hline 191 & 806502.11499751621351505261143 \\
193 & $\underline{806502.11499751621351505261260}$ \\
\hline 1.h.s. of (4.50) & 806502.11499751621351505261016 \\
\hline
\end{tabular}

Table 10. Numerical verification of the characteristic exponent formula (4.50) at $u=0$ and $\hbar=1 / 4$, with increasing number of terms for the lateral Borel resummations on the r.h.s. All the stabilised digits (underlined) are in agreement.

We can now see a clear difference between the TBA equations of [1] and the TBA equations of [5]. The TBA equations of [1] compute the Borel-summable part of the $B$ period, where we have removed the perturbative contribution due to the $\gamma$ function, i.e. they compute (4.49), which is the Borel resummation of $\Pi_{B}^{\text {red }}$ in (4.48). On the contrary, the conformal limit of the GMN TBA equations computes the Borel resummation of the full quantum $B$ period $\Pi_{B}(E, \hbar)$, including the perturbative $\gamma$ function. Since the latter is not Borel summable, the corresponding TBA has discontinuities, as discussed in section 3.2.

In the strong coupling region we have the following relation between the "exact" quantum $A$ period and lateral Borel resummations of quantum periods [15]

$$
\begin{aligned}
2 \cosh \left(\Pi_{A}^{\mathrm{ex}} /(2 \hbar)\right) & =\mathrm{e}^{\frac{1}{2 \hbar} s_{+}\left(\Pi_{A}\right)}+\mathrm{e}^{\frac{1}{2 \hbar} s_{+}\left(\Pi_{A}+2 \Pi_{B}\right)}+\mathrm{e}^{-\frac{1}{2 \hbar} s_{+}\left(\Pi_{A}+2 \Pi_{B}\right)} \\
& =\mathrm{e}^{\frac{1}{2 \hbar} s_{-}\left(\Pi_{A}\right)}+\mathrm{e}^{-\frac{1}{2 \hbar} s_{-}\left(\Pi_{A}\right)}+\mathrm{e}^{\frac{1}{2 \hbar} s_{-}\left(\Pi_{A}+2 \Pi_{B}\right)} .
\end{aligned}
$$

Numerical evidence for this relation is presented in table 10 for the first line of the formula, evaluated at $u=0$ and $\hbar=1 / 4$. The second line of (4.50) can be derived from the Stokes automorphism of quantum periods discussed in section 3.2. In the strong coupling region, $\Pi_{D}=\Pi_{B}+\Pi_{A}$ is Borel summable along the positive real axis, while both the Borel resummations of $\Pi_{B}$ and $\Pi_{A}$ have discontinuities across the positive real axis. The discontinuities for the $B$, and $A$ periods are:

$$
\begin{aligned}
& s_{+}\left(\Pi_{B}\right)-s_{-}\left(\Pi_{B}\right)=2 \hbar \log \left(1+\mathrm{e}^{-\frac{1}{\hbar} s\left(\Pi_{D}\right)}\right), \\
& s_{+}\left(\Pi_{A}\right)-s_{-}\left(\Pi_{A}\right)=-2 \hbar \log \left(1+\mathrm{e}^{-\frac{1}{\hbar} s\left(\Pi_{D}\right)}\right) .
\end{aligned}
$$

Starting from the first line on the right hand side of (4.50), and applying the discontinuity 
formulae, we immediately get the second line

first line

$$
\begin{aligned}
= & \mathrm{e}^{\frac{1}{2 \hbar} s_{+}\left(\Pi_{A}\right)}+\mathrm{e}^{\frac{1}{2 \hbar} s_{+}\left(\Pi_{A}+2 \Pi_{B}\right)}+\mathrm{e}^{-\frac{1}{2 \hbar} s_{+}\left(\Pi_{A}+2 \Pi_{B}\right)} \\
= & \mathrm{e}^{\frac{1}{2 \hbar} s_{-}\left(\Pi_{A}\right)}\left(1+\mathrm{e}^{-\frac{1}{\hbar} s\left(\Pi_{D}\right)}\right)^{-1}+\mathrm{e}^{\frac{1}{2 \hbar} s_{-}\left(\Pi_{A}+2 \Pi_{B}\right)}\left(1+\mathrm{e}^{-\frac{1}{\hbar} s\left(\Pi_{D}\right)}\right) \\
& +\mathrm{e}^{-\frac{1}{2 \hbar} s_{-}\left(\Pi_{A}+2 \Pi_{B}\right)}\left(1+\mathrm{e}^{-\frac{1}{\hbar} s\left(\Pi_{D}\right)}\right)^{-1} \\
= & \left(\mathrm{e}^{\frac{1}{2 \hbar} s_{-}\left(\Pi_{A}\right)}+\mathrm{e}^{-\frac{1}{2 \hbar} s_{-}\left(\Pi_{A}+2 \Pi_{B}\right)}\right)\left(1+\mathrm{e}^{-\frac{1}{\hbar} s\left(\Pi_{D}\right)}\right)^{-1}+\mathrm{e}^{\frac{1}{2 \hbar} s_{-}\left(\Pi_{A}+2 \Pi_{B}\right)}+\mathrm{e}^{-\frac{1}{2 \hbar} s_{-}\left(\Pi_{A}\right)} \\
= & \mathrm{e}^{\frac{1}{2 \hbar} s_{-}\left(\Pi_{A}\right)}+\mathrm{e}^{-\frac{1}{2 \hbar} s_{-}\left(\Pi_{A}\right)}+\mathrm{e}^{\frac{1}{2 \hbar} s_{-}\left(\Pi_{A}+2 \Pi_{B}\right)}=\text { second line. }
\end{aligned}
$$

On the other hand, to derive a similar result for the "exact" quantum $B$ period we can use results on the Fredholm determinant of the modified Mathieu equation, which we present in section 5. Eq. (5.44) then together (4.50) imply that

$$
\begin{aligned}
2 \sinh \left(\Pi_{B}^{\mathrm{ex}} /(2 \hbar)\right) & =\mathrm{e}^{\frac{1}{2 \hbar} s_{+}\left(\Pi_{B}\right)}-\mathrm{e}^{-\frac{1}{2 \hbar} s_{+}\left(\Pi_{B}\right)}-\mathrm{e}^{-\frac{1}{2 \hbar} s_{+}\left(2 \Pi_{A}+3 \Pi_{B}\right)}, \\
& =\mathrm{e}^{\frac{1}{2 \hbar} s_{-}\left(\Pi_{B}\right)}-\mathrm{e}^{-\frac{1}{2 \hbar} s_{-}\left(\Pi_{B}\right)}+\mathrm{e}^{-\frac{1}{2 \hbar} s_{-}\left(2 \Pi_{A}+\Pi_{B}\right)},
\end{aligned}
$$

and we have tested these identities numerically to very high precision.

Summarizing, in the weak coupling region the all-orders WKB quantum $A$ period and the $B$ period (once the gamma function is subtracted) are Borel summable. Their Borel sums agree with the gauge theory expressions of section 4.1. In the strong coupling region, the $A$ and $B$ periods are not Borel summable, although their lateral Borel resummations can be related to the exact quantum $A$ period via (4.50). We finally note that the combination $\Pi_{D}=\Pi_{A}+\Pi_{B}$ is Borel summable in the strong coupling region (only). However, its Borel sum does not agree with the gauge theory expression of section 4.1, namely

$$
s\left(\Pi_{D}\right) \neq \Pi_{A}^{\mathrm{ex}}+\Pi_{B}^{\mathrm{ex}}=2 \pi a+2 \mathrm{i} a_{D},
$$

and one should include additional non-perturbative corrections. We will find the correct formula at the end of section 5.3.

\section{The Fredholm determinant from topological string theory}

Let $\mathrm{O}$ be an operator on $L^{2}(\mathbb{R})$ such that $\mathrm{O}^{-1}$ is of trace class. Then, $\mathrm{O}$ has a discrete spectrum $\left\{E_{n}\right\}_{n \geq 0}$, and its Fredholm determinant

$$
\Xi(E)=\operatorname{det}\left(1-E \mathrm{O}^{-1}\right)=\prod_{n \geq 0}\left(1-\frac{E}{E_{n}}\right)
$$

is an entire function of $E$ whose zeros give the spectrum of O: $\Xi\left(E_{n}\right)=0$ (see e.g. [58] for these and other properties of Fredholm determinants).

The Fredholm determinant contains very rich information about the spectral properties of O. For example, the spectral traces, defined as

$$
Z_{\ell}=\sum_{n \geq 0} \frac{1}{E_{n}^{\ell}},
$$


can be computed by expanding the spectral determinant around $E=0$. Indeed, we have

$$
\Xi(E)=\sum_{N \geq 0}(-E)^{N} Z(N, \hbar)
$$

where

$$
Z(N, \hbar)=\sum_{\left\{m_{\ell}\right\}}^{\prime} \prod_{\ell} \frac{(-1)^{(\ell-1) m_{\ell}} Z_{\ell}^{m_{\ell}}}{m_{\ell} ! \ell^{m_{\ell}}},
$$

and the ' means that the sum is over the integers $m_{\ell}$ satisfying the constraint

$$
\sum_{\ell} \ell m_{\ell}=N
$$

From the quantities $Z(N, \hbar)$ (which were called fermionic spectral traces in [2]) one can extract the conventional spectral traces (5.2).

Although Fredholm determinants are central objects in spectral theory, it is difficult to obtain explicit expressions for them. It is easy to show (see for instance [59]) that the inverse of the modified Mathieu operator (2.14) is of trace class. Therefore, the Fredholm determinant is well-defined, and it is an interesting question to find an explicit, closed form expression for this quantity.

In recent years it was discovered [2,3,60-62] that, by using topological string tools, it is possible to obtain explicitly expression for Fredholm determinants of operators arising in the context to quantum mirror curves. We will refer to this relationship as the TS/ST correspondence. As explained in section 5.1, the modified Mathieu operator (2.14) can be related, upon a suitable limiting procedure, to the quantum mirror curve of local $\mathbb{F}_{0}$. Therefore we can study (5.1) within the context of topological string theory and in particular, by using $[2,18]$, we can deduce an explicit, closed form expression for the Fredholm determinant of the modified Mathieu operator. We will first state the main result and then explain how to derive it within topological string theory. We also present several independent tests of our result, including an interesting connection to the TBA system of [19].

\subsection{A closed formula and its derivation}

By using the approach of $[2,18]$ we find the following expression for the spectral determinant of $(2.14)^{6}$

$$
\Xi(E)=A(\hbar)\left(\sinh \left(\frac{\Pi_{A}^{\mathrm{ex}}(E, \hbar)}{2 \hbar}\right)\right)^{-1} \cosh \left(\frac{1}{2 \hbar} \Pi_{B}^{\mathrm{ex}}(E, \hbar)\right),
$$

where $\Pi_{A, B}^{\text {ex }}(E, \hbar)$ are given by $(4.18)$ and $A(\hbar)$ is an $u$-independent constant which can be fixed from $\Xi(0)=1$, namely,

$$
A(\hbar)=\sinh \left(\frac{\Pi_{A}^{\mathrm{ex}}(0, \hbar)}{2 \hbar}\right)\left(\cosh \left(\frac{1}{2 \hbar} \Pi_{B}^{\mathrm{ex}}(0, \hbar)\right)\right)^{-1} .
$$

\footnotetext{
${ }^{6}$ While presenting these results at the conference Irregular singularities in Quantum Field Theory (http://irregular.rd.ciencias.ulisboa.pt/conference), S. Lukyanov informed us that he had independently derived this result [63] by using completely different methods.
} 
From this expression we can read off explicit formulae for the spectral traces. We find for instance

$$
Z_{1}=-\frac{1}{2 \hbar}\left(\partial_{E} \Pi_{B}^{\mathrm{ex}}(0, \hbar) \tanh \left(\frac{\Pi_{B}^{\mathrm{ex}}(0, \hbar)}{2 \hbar}\right)-\operatorname{coth}\left(\frac{\Pi_{A}^{\mathrm{ex}}(0, \hbar)}{2 \hbar}\right) \partial_{E} \Pi_{A}^{\mathrm{ex}}(0, \hbar)\right),
$$

as well as

$$
\begin{aligned}
4 \hbar^{2} Z_{2}= & 2 \hbar \partial_{E}^{2} \Pi_{A}^{\mathrm{ex}}(0, \hbar) \operatorname{coth}\left(\frac{\Pi_{A}^{\mathrm{ex}}(0, \hbar)}{2 \hbar}\right)-\left(\partial_{E} \Pi_{A}^{\mathrm{ex}}(0, \hbar)\right)^{2} \operatorname{csch}^{2}\left(\frac{\Pi_{A}^{\mathrm{ex}}(0, \hbar)}{2 \hbar}\right) \\
& -\operatorname{sech}^{2}\left(\frac{\Pi_{B}^{\mathrm{ex}}(0, \hbar)}{2 \hbar}\right)\left(\hbar \partial_{E}^{2} \Pi_{B}^{\mathrm{ex}}(0, \hbar) \sinh \left(\frac{\Pi_{B}^{\mathrm{ex}}(0, \hbar)}{\hbar}\right)+\left(\partial_{E} \Pi_{B}^{\mathrm{ex}}(0, \hbar)\right)^{2}\right) .
\end{aligned}
$$

Note that in order to calculate $\Pi_{A}^{\mathrm{ex}}(0, \hbar)$ we have to use (4.37) or (4.38), so the above formula tests as well the analytic continuation of instanton calculus beyond the semiclassical region. ${ }^{7}$

The explicit formula (5.6) can be extended to the family of operators considered in [18], see appendix A for more details.

Let us now explain how to derive (5.6) from the TS/ST correspondence of $[2,3]$. The relevant $\mathrm{CY}$ geometry is the canonical bundle over $\mathbb{F}_{0}$, also known as resolved $Y^{2,0}$ singularity. The corresponding quantum mirror curve is

$$
\mathrm{O}-\kappa=(R \Lambda)^{2}\left(\mathrm{e}^{\mathrm{x}}+\mathrm{e}^{-\mathrm{x}}\right)-\kappa+\mathrm{e}^{\mathrm{p}}+\mathrm{e}^{-\mathrm{p}}, \quad[\mathrm{x}, \mathrm{p}]=\mathrm{i} \hbar .
$$

According to the TS/ST correspondence we have

$$
\operatorname{det}\left(1-\kappa \mathrm{O}^{-1}\right)=\sum_{m \in \mathbb{Z}} \exp [\mathrm{J}(\mu+\mathrm{i} \pi+2 \pi \mathrm{i} m, R \Lambda, \hbar)], \quad \kappa=\mathrm{e}^{\mu},
$$

where $\mathrm{J}(\mu, \xi, \hbar)$ is the grand potential of the resolved $Y^{2,0}$ singularity as defined in [18], section 5.1. The expression (5.6) is obtained by implementing the geometric engineering limit $[37,64]$ in $(5.11)$. More precisely, we consider the limit

$$
p \rightarrow R p, \quad \hbar \rightarrow R \hbar, \quad \kappa=2+E R^{2}+\mathcal{O}\left(R^{3}\right), \quad R \rightarrow 0 .
$$

This has to be done carefully since both sides of (5.11) diverge, therefore they need to be properly regularized. For that it is convenient to study the trace of the resolvent

$$
G_{\mathrm{O}}(\kappa)=\frac{\mathrm{d}}{\mathrm{d} \kappa} \log \operatorname{det}\left(1-\kappa \mathrm{O}^{-1}\right)=\operatorname{Tr}\left(\frac{1}{\kappa-\mathrm{O}}\right),
$$

rather than the spectral determinant. It is easy to see that in the limit (5.12) one has

$$
G_{\mathrm{O}}(\kappa) \rightarrow \frac{1}{R^{2}} G(E), \quad G(E)=\operatorname{Tr}\left(\frac{1}{E-\mathrm{H}}\right),
$$

where $\mathrm{H}$ is the modified Mathieu operator in (2.14). Likewise the limit (5.12) can be implemented on the r.h.s of (5.11) in a quite straightforward way by following [18], section 5.2 and by using the identity (3.9) in [65]. The overall divergent piece $R^{-2}$ cancels and we find

$$
G(E)=-\frac{\partial_{E} a}{\hbar}\left(\partial_{a}^{2} F_{\mathrm{NS}} \tanh \left(\frac{\partial_{a} F_{\mathrm{NS}}}{\hbar}\right)+\pi \operatorname{coth}\left(\frac{a \pi}{\hbar}\right)\right) .
$$

By integrating w.r.t. $E$ we obtain (5.6), where $A(\hbar)$ is an integration constant.

\footnotetext{
${ }^{7}$ In evaluating the derivative of the periods w.r.t. $E$, we used the quantum Matone relation (4.14) and instanton calculus.
} 


\subsection{Tests of our formula}

We will now test the expression (5.6) in several ways.

A first simple test is that the zeros of $\Xi(E)$ give the correct spectrum of the modified Mathieu operator. This should be expected from the general results of [18], but it is instructive to check it explicitly. The zeros correspond to the vanishing of the cosh in (5.6), and by using (4.18) we find,

$$
\partial_{a} F_{\mathrm{NS}}(a, \hbar)=\hbar \pi\left(n+\frac{1}{2}\right),
$$

which is the exact quantization condition obtained from the conjecture in [1], subsequently proved in [56].

Another check can be obtained by comparing our expression to the asymptotics of Fredholm determinants obtained in $[20,21,66]$ by using the all-orders WKB method. This asymptotic expansion is valid when $E<0$, where the Fredholm determinant is not oscillatory. In order to write down the asymptotics, we need some ingredients. Let

$$
G(E)=\sum_{n=0}^{\infty} \frac{1}{E-E_{n}}
$$

be the trace of the resolvent, and

$$
T(E)=\frac{1}{2} \int_{\mathbb{R}} \frac{\mathrm{d} x}{\sqrt{2 \Lambda^{2} \cosh (x)-E}}
$$

the transit time. We also need the formal power series in $\hbar$

$$
b(E)=\exp \left\{\mathrm{i} \sum_{n \geq 1} \hbar^{2 n-1} \int_{\mathbb{R}} p_{2 n}(x, E) \mathrm{d} x\right\},
$$

where the functions $p_{2 n}(x, E)$ are the ones appearing in the solution to the all-orders WKB method in (2.4). Let us now define $\kappa=-E$, which will be taken to be positive. It is convenient to introduce the functions

$$
\widetilde{G}(\kappa)=G(-\kappa), \quad \widetilde{\Xi}(\kappa)=\Xi(-\kappa), \quad \widetilde{b}(\kappa)=b(-\kappa), \quad \widetilde{T}(\kappa)=T(-\kappa) .
$$

Then, one has the following small $\hbar$ asymptotics,

$$
\widetilde{\Xi}(\kappa) \sim \widetilde{b}(\kappa) \exp \left\{\frac{1}{\hbar} \int_{0}^{\kappa} \widetilde{T}\left(\kappa^{\prime}\right) \mathrm{d} \kappa^{\prime}-\int_{0}^{\infty}\left(\widetilde{G}\left(\kappa^{\prime}\right)+\frac{1}{\hbar} \widetilde{T}\left(\kappa^{\prime}\right)\right) \mathrm{d} \kappa^{\prime}\right\} .
$$

The second term in the exponent is independent of $\kappa$ but depends on $\hbar$. We note that all the integrals involved in this expression are well defined precisely because $E$ is negative. The very first terms in the asymptotics can be easily worked out, and one finds ${ }^{8}$

$$
\log \Xi(-\kappa) \sim-\frac{\mathrm{i}}{2 \hbar} \Pi_{B}^{(0)}(\kappa)+\frac{\mathrm{i} \hbar}{2} \Pi_{B}^{(1)}(\kappa)+\cdots, \quad \kappa>0,
$$

\footnotetext{
${ }^{8}$ This agrees with an unpublished calculation of Y. Hatsuda, who obtained the same result by considering the semiclassical expansion of the spectral traces.
} 
up to $\kappa$-independent terms. Note that the sign in the subleading correction is the opposite one to what one finds in the WKB expansion of the quantum $B$ period.

Let us now compare this result to the exact expression for the spectral determinant (5.6), which can be written as

$$
\Xi(E)=A(\hbar)\left(\sinh \left(\frac{\pi a}{\hbar}\right)\right)^{-1} \cos \left(\frac{1}{\hbar} \frac{\partial F_{\mathrm{NS}}}{\partial a}\right) .
$$

From the explicit expression (4.28) it is easy to see that, when $E$ is negative, $a$ is purely imaginary. More precisely, one has

$$
a(-\kappa, \hbar)=\mathrm{i} \alpha, \quad \alpha=a(\kappa, \mathrm{i} \hbar),
$$

and we take $\alpha>0$ for definiteness. In addition,

$$
\partial_{a} F_{\mathrm{NS}}^{\mathrm{inst}}(a, \hbar)=\mathrm{i} \partial_{\alpha} F_{\mathrm{NS}}^{\mathrm{inst}}(\alpha, \mathrm{i} \hbar) .
$$

By using the explicit expression (4.16) and standard identities for the $\Gamma$ function, we find

$$
\begin{aligned}
\frac{\Xi(-\kappa)}{A(\hbar)}= & \frac{1}{2 \pi} \Gamma\left(1+\frac{\alpha}{\hbar}\right) \Gamma\left(\frac{\alpha}{\hbar}\right) \exp \left[\frac{\alpha}{\hbar} \log \left(\frac{\hbar^{2}}{\Lambda^{2}}\right)+\frac{1}{\hbar} \partial_{\alpha} F_{\mathrm{NS}}^{\text {inst }}(\alpha, \mathrm{i} \hbar)\right] \\
& +\frac{1}{2 \pi} \Gamma\left(1-\frac{\alpha}{\hbar}\right) \Gamma\left(-\frac{\alpha}{\hbar}\right) \exp \left[-\frac{\alpha}{\hbar} \log \left(\frac{\hbar^{2}}{\Lambda^{2}}\right)-\frac{1}{\hbar} \partial_{\alpha} F_{\mathrm{NS}}^{\text {inst }}(\alpha, \mathrm{i} \hbar)\right] .
\end{aligned}
$$

The term in the second line gives an exponentially small correction to the leading asymptotics. The small $\hbar$ asymptotics of the quantity in the first line is given by

$$
\begin{aligned}
& \log \left[\frac{1}{2 \pi} \Gamma\left(1+\frac{\alpha}{\hbar}\right) \Gamma\left(\frac{\alpha}{\hbar}\right)\right]+\frac{\alpha}{\hbar} \log \left(\frac{\hbar^{2}}{\Lambda^{2}}\right)+\frac{1}{\hbar} \partial_{\alpha} F_{\mathrm{NS}}^{\mathrm{inst}}(\alpha, \mathrm{i} \hbar) \\
& \sim-\frac{\mathrm{i}}{2 \hbar} \sum_{n \geq 0}(-1)^{n} \Pi_{B}^{(n)}(\kappa) \hbar^{2 n} .
\end{aligned}
$$

We have used that, due to (5.24) and (5.25), the quantum period is evaluated at $-E$, where $E=-\kappa<0$, and we have to change $\hbar \rightarrow \mathrm{i} \hbar$. The result is in agreement with the WKB asymptotics obtained in (5.22).

A more precise test of (5.6) can be made by comparing the analytical formulae for the spectral traces with numerical results. These are obtained by calculating the spectrum of $\mathrm{H}$ with standard techniques. An example of such a comparison is shown in table 11.

We finally note that $\Xi(E)$ is an entire function of $E$. In particular, the would-be singularities due to the denominator of (5.23) or to the Gamma functions in (4.16) must cancel in the end. This leads in turn to constraints on the form of the singularities of $F_{\mathrm{NS}}^{\text {inst }}$, which might be testable against the results in [67] (see also [68]).

\subsection{Comparison to Zamolodchikov's TBA equation}

An additional test of our formula (5.6) comes from a comparison with [19]. Inspired by the ODE/IM correspondence [69, 70], Zamolodchikov found in [19] a TBA equation which computes precisely the spectral determinant (5.6). Let us state the main result 


\begin{tabular}{|ll|}
\hline$N_{b}$ & $Z_{2}$ \\
\hline 2 & $\underline{0.004794786} 11468342466$ \\
4 & $\underline{0.004794786073913} 81196$ \\
6 & 0.00479478607391375025 \\
\hline Num & 0.00479478607391375025 \\
\hline
\end{tabular}

Table 11. The second spectral trace $Z_{2}$ as computed from (5.9) for $\hbar=3 \pi$ and $\Lambda=1$. The number $N_{b}$ means that we truncate the series (4.13) at order $\Lambda^{4 N_{b}}$. The last line gives the numerical result obtained from the spectrum of $\mathrm{H}$.

of [19], referring to appendix B for more details. Let $\epsilon(\theta, P)$ be a solution of the TBA equation (3.69) but with the boundary condition at $\theta \rightarrow-\infty$ given by

$$
\epsilon(\theta, P) \sim 8 P \theta-2 C(P), \quad \theta \rightarrow-\infty, \quad P>0
$$

where $C(P)$ is written down in (B.15). Let us now introduce the function

$$
X(\mu, P)=\exp [-\epsilon(\theta, P) / 2]
$$

where $\mu$ is related to $\theta$ by

$$
\mu=\mathrm{e}^{2 \theta}\left(\frac{\Gamma\left(\frac{1}{4}\right)^{2}}{16 \sqrt{\pi}}\right)^{2} .
$$

Then, according to [19], the spectral determinant of the modified Mathieu operator (2.14) is given by

$$
\Xi(E)=\frac{X(\mu, P)}{X(\mu, 0)},
$$

where the parameters $\Lambda, E$ and $\hbar$ of the operator are related to the parameters appearing in $X(\mu, P)$ by

$$
\mu=\Lambda^{2} \hbar^{-2}, \quad P^{2}=-E \hbar^{-2} .
$$

If we compare the result of [19] with ours we should have (by using the dictionary (5.32))

$$
\frac{X(\mu, P)}{X(\mu, 0)}=A(\hbar)\left(\sinh \left(\frac{\Pi_{A}^{\mathrm{ex}}(E, \hbar)}{2 \hbar}\right)\right)^{-1} \cosh \left(\frac{1}{2 \hbar} \Pi_{B}^{\mathrm{ex}}(E, \hbar)\right) .
$$

In order to find the relation between the two normalization constants $X(\mu, 0)$ and $A(\hbar)$, it is useful to first derive the asymptotic behavior (5.28) from our expression (5.6). We need to expand around small $\Lambda \hbar^{-1}$ and take $u<0$, which means that $a$ is imaginary, as discussed in (5.24). In this regime, and by using (4.18), we have

$$
\begin{aligned}
& \left(\sinh \left(\frac{\pi a}{\hbar}\right)\right)^{-1} \cosh \left(\frac{\mathrm{i}}{\hbar} \partial_{a} F_{\mathrm{NS}}(a, \hbar)\right) \\
& \approx 2^{-1} \pi^{-1}\left(\left(\frac{\Lambda}{\hbar}\right)^{-2 s} \Gamma(1+s) \Gamma(s)+\left(\frac{\Lambda}{\hbar}\right)^{2 s} \Gamma(1-s) \Gamma(-s)\right),
\end{aligned}
$$




\begin{tabular}{|ll|}
\hline$N_{b}$ & $\log \left(\left(\sinh \left(\frac{\pi a}{\hbar}\right)\right)^{-1} \cosh \left(\frac{\mathrm{i}}{\hbar} \partial_{a} F_{\mathrm{NS}}(a, \hbar)\right)\right)$ \\
\hline 2 & $\underline{11.360025317439438}$ \\
4 & $\underline{11.360025299112863}$ \\
6 & 11.360025299117259 \\
\hline TBA & 11.360025299117 \\
\hline
\end{tabular}

Table 12. The un-normalized spectral determinant as computed by using instanton counting and by solving numerically the TBA (3.69) with (5.28). We use $P^{2}=-E=5, \mu=\Lambda^{2}=$ $\left(\Gamma(1 / 4)^{2} /(16 \sqrt{\pi})\right)^{2}$, and $\hbar=1$. The number $N_{b}$ means that we truncate the series (4.13) at order $\Lambda^{4 N_{b}}$.

where

$$
s=-\mathrm{i} \frac{a}{\hbar}=\frac{\alpha}{\hbar}>0 .
$$

By using (4.14) we have

$$
E \approx a^{2} / 4
$$

and therefore

$$
P=s / 2>0 .
$$

Hence we can neglect the second term in the r.h.s. of (5.34). It follows from (5.32) that (5.34) agrees precisely with (5.28). In particular this means that the two normalization constants are identified and we have

$$
X(\mu, P)=\left(\sinh \left(\frac{\Pi_{A}^{\mathrm{ex}}(E, \hbar)}{2 \hbar}\right)\right)^{-1} \cosh \left(\frac{1}{2 \hbar} \Pi_{B}^{\mathrm{ex}}(E, \hbar)\right) .
$$

We test this equality by solving numerically the TBA equation (3.69) with the boundary condition (5.28). Some results are given in table 12. We find perfect agreement.

An important spinoff of this comparison is that our result (5.6) provides an analytic, closed form solution to the TBA equation of [19]. This also has the following consequence. When we derived the Fredholm determinant from the topological string perspective, and due to our regularization procedure, we generated an integration constant $A(\hbar)$ whose explicit expression is given in (5.7). Given the identity (5.38) between our Fredholm determinant and the solution to the Zamolodchikov's TBA, we expect $A(\hbar)$ to be computed by the integral equation (3.69) at $P=u=0$. More precisely we expect

$$
2 \log A(\hbar)=\epsilon(\theta, P=0),
$$

where we used the dictionary (5.32). For $P=0$ the asymptotic condition (5.28) does not make sense, strictly speaking. Nevertheless, we can derive the appropriate asymptotic condition for the TBA at $P=0$ by using our analytic expression (5.38). We find that, as $\theta \rightarrow-\infty$,

$$
\epsilon(\theta, P=0) \sim-2 \log \left(-2\left(\theta+\gamma_{\text {Euler }}\right)+\log (\pi)-4 \log \left(\Gamma\left(\frac{5}{4}\right)\right)\right) .
$$


This is precisely the boundary condition used in section 3.3, equation (3.74). One can now check (5.39) numerically. For instance, by solving the TBA of section 3.3 we find

$$
\left.\epsilon(\theta, P=0)\right|_{\theta=-1}=0.51888 \cdots
$$

Likewise, by using instanton counting, and in particular (4.18) and (5.7), we have $(\Lambda=1)$

$$
2 \log \left(A\left(\frac{16 \mathrm{e} \sqrt{\pi}}{\Gamma\left(\frac{1}{4}\right)^{2}}\right)\right)=0.51887965286656 \cdots
$$

We have 5 matching digits which is consistent with the precision achieved with the TBA equation.

This discussion provides an additional result along the lines of what we obtained in section 4.2. As we discussed in section 3.3, the function $\epsilon(\theta)$ with the boundary condition (3.74) computes the dyonic period $\Pi_{D}(0, \hbar)$. As pointed out in section 4.2 , such period is Borel summable, and we can indeed test that its Borel resummation agrees with (5.7), namely

$$
\exp \left(\frac{1}{2 \hbar} s\left(\Pi_{D}\right)(0, \hbar)\right)=\left.\frac{\sinh \left(\frac{\pi a}{\hbar}\right)}{\cosh \left(\frac{\mathrm{i}}{\hbar} \partial_{a} F_{\mathrm{NS}}(a, \hbar)\right)}\right|_{a=a(0, \hbar)}=\frac{\sinh \left(\frac{1}{2 \hbar} \Pi_{A}^{\mathrm{ex}}(0, \hbar)\right)}{\cosh \left(\frac{1}{2 \hbar} \Pi_{B}^{\mathrm{ex}}(0, \hbar)\right)}
$$

We have verified this identity numerically. In addition we have tested that (5.43) also holds for other values of $u$ in the strong coupling region, and we conjecture that, for $u \in[-1,1]$, one has

$$
\exp \left(\frac{1}{2 \hbar} s\left(\Pi_{D}\right)(u, \hbar)\right)=\frac{\sinh \left(\frac{1}{2 \hbar} \Pi_{A}^{\mathrm{ex}}(u, \hbar)\right)}{\cosh \left(\frac{1}{2 \hbar} \Pi_{B}^{\mathrm{ex}}(u, \hbar)\right)} .
$$

\section{On the modified Mathieu operator and Painlevé $\mathrm{III}_{3}$}

It was observed by many authors $[63,71,72]$ that the movable poles of Painlevé $\mathrm{III}_{3}$ are somehow related to Mathieu functions. In particular in [73], based on [65], it was observed that the zeros of the Painlevé $\mathrm{III}_{3} \tau$ function compute the spectrum of modified Mathieu (with a suitable dictionary). From the view point of the TS/ST correspondence [2] this connection comes naturally since both systems arise as limiting cases of this duality. In particular, the modified Mathieu operator arises in the standard geometric engineering limit $[18,55,74]$, while Painlevé $\mathrm{III}_{3}$ arises in the dual geometric engineering limit considered in [65].

In this section we prove the connection between the zeros of the Painlevé $\mathrm{III}_{3} \tau$ function and the spectrum of the modified Mathieu operator by using [75]. From the CFT perspective this is a connection between Liouville conformal blocks at $c=1$ and $c=\infty$. We proceed as follows. First we write the Painlevé $\mathrm{III}_{3} \tau$ function as [76-78]

$$
\tau(\Lambda, a, \eta, \hbar)=\sum_{n \in \mathbb{Z}} \mathrm{e}^{4 \pi \mathrm{i} n \eta} \exp \left(F^{\mathrm{SD}}(a+2 \mathrm{i} \hbar n, \hbar, \Lambda)\right)
$$


where

$$
\begin{aligned}
\exp \left(F^{\mathrm{SD}}(a, \hbar, \Lambda)\right)= & \left(\frac{\Lambda}{\hbar}\right)^{-\frac{a^{2}}{\hbar^{2}}} \frac{1}{G\left(1-\mathrm{i} \frac{a}{\hbar}\right) G\left(1+\mathrm{i} \frac{a}{\hbar}\right)} \\
& \times\left(1-\frac{2 \Lambda^{4}}{a^{2} \hbar^{2}}+\frac{\Lambda^{8}\left(2 a^{2}-\hbar^{2}\right)}{a^{2} \hbar^{2}\left(a^{2}+\hbar^{2}\right)^{2}}+\mathcal{O}\left(\Lambda^{12}\right)\right)
\end{aligned}
$$

is the so-called four dimensional Nekrasov partition function in the selfdual $\Omega$ background [45] (namely, the equivariant parameters are $\epsilon_{1}=-\epsilon_{2}=\hbar$ ). The parameters $(a, \eta)$ in (6.1) play the role of initial conditions while $\Lambda$ is the time. We are interested in the case in which

$$
\eta=0
$$

We now recall the result of [75], where it was demonstrated that, in the NS limit, the Nakajima-Yoshioka blowup equations for SU(2) pure SYM [79-81] can be written as ${ }^{9}$

$$
\sum_{n \in \mathbb{Z}} \exp \left(\mathrm{i} n \pi+F^{\mathrm{SD}}(a+2 \mathrm{i} \hbar n+\mathrm{i} \hbar, \hbar, \Lambda)-2 \mathrm{i} n \hbar^{-1} \frac{\partial}{\partial a} F_{\mathrm{NS}}(a, \hbar)\right)=0 .
$$

Finally, we use the quantization condition for the modified Mathieu operator in the NS form (5.16). It then follows from (6.4) that, if a value of $a$ satisfies this exact quantization condition, one finds a vanishing condition for the tau function of Painlevé $\mathrm{III}_{3}$, namely

$$
\tau(\Lambda, a+\mathrm{i} \hbar, 0, \hbar)=\sum_{n \in \mathbb{Z}} \exp \left(F^{\mathrm{SD}}(a+2 \mathrm{i} \hbar n+\mathrm{i} \hbar, \hbar, \Lambda)\right)=0
$$

Notice that we think of (5.16) and (6.5) as quantization conditions for the variable $a$. In order to obtain the spectrum of modified Mathieu one has to use the quantum Matone relation (4.14).

\section{Conclusions}

In this paper we have used non-perturbative techniques inspired by supersymmetric gauge theory and topological string theory to study the quantization of the Seiberg-Witten curve of $\mathcal{N}=2, \mathrm{SU}(2)$ super Yang-Mills theory, which gives the modified Mathieu operator. On the one hand, building upon [5-8], we have obtained integral equations for the Borel resummation of the quantum periods obtained with the all-orders WKB method. These equations predict as well the resurgent structure of these periods, and in particular their Stokes discontinuities. The results obtained in this way have been tested against calculations in the WKB method to very high order. We have also clarified the relation between these Borel-resummed quantum periods and the "exact" quantum periods given by instanton calculus (in the NS limit). On the other hand, we have used the TS/ST correspondence

\footnotetext{
${ }^{9}$ Strictly speaking this is the four-dimensional limit of [75]. This type of expressions first appeared in [82] as compatibility conditions between the exact quantization conditions of $[2,3]$ and those of $[55,83,84]$. A different connection between blowup and Painlevé equations was used in [85-87] to prove the so-called Kiev formula $[76,88]$ or its q-deformed version [89, 90].
} 
of $[2,3]$ to obtain a closed formula for the spectral determinant of the modified Mathieu operator, and we have compared this formula to previous results by Zamolodchikov.

Our results raise several issues. An important problem concerns the relation between the TBA equations obtained in the context of SW theory, and the analytic bootstrap program first proposed in [21] and reloaded in [8,9]. In the TBA equations obtained in $[7,8]$ for quantum mechanics with polynomial potentials, one only needs the boundary condition associated to the classical behavior (i.e. at $\hbar \rightarrow 0$, or equivalently at $\theta \rightarrow \infty$ ). The boundary behavior when $\theta \rightarrow-\infty$ is fixed by the integral equations. As pointed out already in [7] and further discussed in section 3.3 of this paper, the integral equations for the modified Mathieu operator admit many possible boundary conditions at $\theta \rightarrow-\infty$, and one needs additional information to fix them. One can use the quantum Matone relation and the first quantum correction to the periods to obtain additional constraints. However, it seems clear from the study of this example that the analytic bootstrap might require additional asymptotic information to determine uniquely the resummed quantum periods. As suggested in [7], one might obtain the appropriate boundary conditions by first solving the full TBA equations of [5] (before taking the conformal limit) and then implementing the conformal limit directly on the solution.

Another problem that should be discussed more carefully is how to solve efficiently the TBA equations to compute the Borel resummed quantum periods. In particular, we should understand in detail how to solve the infinite tower of TBA equations appearing in the weak coupling region.

It would be very interesting to extend the techniques developed in this paper to quantum mirror curves. This would provide a relation between BPS states in local CY threefolds (studied for example in [91]) and the resurgent properties of the corresponding quantum periods. Work along this direction has been already done in [92, 93]. Another interesting class of quantum curves which could be studied with our methods is the one given by quantum A-polynomials of knots (see e.g. [94]). In this case, the resurgent properties should be closely related to the resurgent properties of Andersen-Kashaev invariants [95], which have been considered in [96-98]. They might correspond to BPS states in the supersymmetric dual obtained with the $3 \mathrm{~d} / 3 \mathrm{~d}$ correspondence of [99].

Another intriguing point is the following. Based on previous works $[7,8]$, we have shown that the conformal limit of the GMN TBA equations encode in a precise way the NS limit of the Omega background for the pure $\mathrm{SU}(2)$ theory. On the other hand it is interesting to observe that, as pointed out in [65], there is another set of TBA equations which computes the selfdual limit of the Omega background. The latter was obtained by Zamolodchikov in [100], see also [101]. Interestingly also such TBA can be obtained from [5] upon a suitable limiting procedure. It would be interesting to investigate more concretely if and how the full TBA equations of [5] encode the full Omega background. Work along this direction was performed in [102].

In addition it should be possible to extend the results of section 6 to Painlevé $\mathrm{III}_{2}, \mathrm{III}_{1}$, $\mathrm{V}$ and VI. In these cases, the rôle of the modified Mathieu operator is replaced by the quantum SW curve of $\mathrm{SU}(2)$ gauge theory with $N_{f}=1,2,3,4$ flavours, respectively. In particular, for $N_{f}=4$ one should recover the connection between Painlevé VI and the 
Heun operator [103] (see also [104]). Likewise the spectrum of the Calogero-Moser system should make contact with the $\tau$ function describing the isomonodromic deformations on the torus [105]. The details will appear somewhere else [106].

The situation for Painlevé I, II, IV is more subtle since these correspond to ArgyresDouglas theories of type $H_{0}, H_{1}, H_{2}$, respectively [107]. At present we do not know how to write Nakajima-Yoshioka blowup equations for these theories. Nevertheless, it should be possible to connect the NS limit to the selfdual limit of the $\Omega$ background also in these theories, since the $H_{i}$ theories can be derived from $\mathrm{SU}(2)$ gauge theories with $N_{f}=1,2,3$ upon a suitable limiting procedure [108, 109]. By following [110, 111], such connection would provide a relation between the exact spectrum of the quantum SW curves underlying the $H_{0}, H_{1}, H_{2}$ theories, and Painlevé I, II, IV tau functions. Note that the quantum SW curve of the $H_{0}$ and $H_{1}$ theories correspond to the cubic and quartic oscillators, respectively. Connections between Painlevé equations and the above quantum mechanical systems have been observed in [112-115].

\section{Acknowledgments}

We would like to thank Vladimir Bazhanov, Katsushi Ito, Davide Gaiotto, Lotte Hollands, Amir-Kian Kashani-Poor, Sergei Lukyanov, Davide Masoero, Gregory Moore, Andy Neitzke, Stefano Negro, Nikita Nekrasov, Hongfei Shu, Leon Takhtajan, Roberto Tateo and Peter Wittwer for useful discussions and correspondence. The work of J.G. and M.M. is supported in part by the Fonds National Suisse, subsidy 200021-175539 and by the NCCR 51NF40-182902 "The Mathematics of Physics" (SwissMAP).

\section{A The four dimensional $\mathrm{SU}(N)$ spectral determinant}

In this appendix we explain how the exact formula (5.6) can be extended to the family of operators studied in [18]. These operators have the form,

$$
\mathrm{H}_{N}=\Lambda^{N}\left(\mathrm{e}^{\mathrm{p}}+\mathrm{e}^{-\mathrm{p}}\right)+\sum_{k=0}^{N-1}(-1)^{k} \mathrm{x}^{N-k} h_{k}, \quad[\mathrm{x}, \mathrm{p}]=\mathrm{i} \hbar,
$$

where $N \geq 2$ is a positive integer and we set $h_{0}=1, h_{1}=0$. They can be regarded as deformations of the standard non-relativistic Schrödinger operators with a polynomial potential. When $N$ is even, they have a discrete spectrum and their inverses are of trace class. When $N$ is odd, one can perform a standard analytic continuation and obtain a discrete spectrum of resonances, as explained in [18]. In both cases, one can define a Fredholm determinant as

$$
\Xi_{N}\left(h_{2}, \cdots, h_{N}\right)=\operatorname{det}\left(1+\frac{h_{N}(-1)^{N}}{\mathrm{H}_{N}}\right) .
$$

Here, $h_{2}, \cdots, h_{N-1}$ are the moduli appearing in the potential, while $(-1)^{N-1} h_{N}$ can be identified with the energy and is the standard auxiliary variable appearing in the definition of Fredholm determinants. 
As explained in [18], we can engineer the following operator from the quantum mirror curve to the $Y^{N, 0}$ geometry. We follow [18] and define

$$
\gamma=\frac{1}{2} \sum_{i=1}^{N}(-1)^{i-1} \boldsymbol{e}_{i}
$$

where $\boldsymbol{e}_{i}$ are the weights of the fundamental representation of $\mathrm{SU}(N)$. We denote by

$$
\mathcal{W}_{N} \cdot \gamma=\left\{w(\gamma): w \in \mathcal{W}_{N}\right\}
$$

the Weyl orbit of $\boldsymbol{\gamma}$, and we introduce

$$
\boldsymbol{a}=\sum_{j=1}^{N-1} a_{i} \boldsymbol{\lambda},
$$

where $\left\{\boldsymbol{\lambda}_{i}\right\}_{i=1, \cdots, N-1}$ are the fundamental weights of $\mathrm{SU}(N)$. The quantities $a_{i}$ are related to the parameters $h_{i}$ in (A.1) by using the four dimensional mirror maps or quantum Matone relations (see for instance eqs. (3.95)-(3.107) in [18] and reference therein). For instance, we have

$$
\begin{aligned}
& h_{2}(\boldsymbol{a} ; \hbar)=\lim _{\epsilon_{2} \rightarrow 0}\left(-\frac{1}{Z} \sum_{\boldsymbol{Y}}\left((-1)^{N} \Lambda^{2 N}\right)^{\ell(\boldsymbol{Y})} \mathcal{C}_{2}(\boldsymbol{a}, \boldsymbol{Y}) \mathcal{Z}_{\boldsymbol{Y}}\right), \\
& h_{3}(\boldsymbol{a} ; \hbar)=\lim _{\epsilon_{2} \rightarrow 0}\left(\frac{2}{Z} \sum_{\boldsymbol{Y}}\left((-1)^{N} \Lambda^{2 N}\right)^{\ell(\boldsymbol{Y})} \mathcal{C}_{3}(\boldsymbol{a}, \boldsymbol{Y}) \mathcal{Z}_{\boldsymbol{Y}}\right),
\end{aligned}
$$

where $\mathcal{Z}_{\boldsymbol{Y}}, Z$ are defined in (4.9) and (4.8), and $\boldsymbol{Y}$ is a vector of Young diagrams as in (4.4). Moreover,

$$
\begin{aligned}
\mathcal{C}_{2}(\boldsymbol{a}, \boldsymbol{Y})= & \frac{1}{2} \sum_{I=1}^{N} \alpha_{I}^{2}-\mathrm{i} \hbar \epsilon_{2} \ell(\boldsymbol{Y}), \\
\mathcal{C}_{3}(\boldsymbol{a}, \boldsymbol{Y})= & \mathrm{i} \hbar \epsilon_{2}\left(\frac{\mathrm{i} \hbar+\epsilon_{2}}{2} \ell(\boldsymbol{Y})+\mathrm{i} \hbar \sum_{I=1}^{N} c_{2}\left(Y_{I}^{t}\right)+\epsilon_{2} \sum_{I=1}^{N} c_{2}\left(Y_{I}\right)-\sum_{I=1}^{N} \alpha_{I} \ell\left(Y_{I}\right)\right) \\
& +\frac{1}{6} \sum_{I=1}^{N} \alpha_{I}^{3},
\end{aligned}
$$

where $\ell(\boldsymbol{Y})$ is defined in (4.6) and we use

$$
c_{2}(Y)=\frac{1}{2} \sum_{i \geq 1} y_{i}\left(y_{i}-1\right) .
$$

We also denote

$$
a_{i}=\alpha_{i}-\alpha_{i+1}, \quad i=1, \cdots, N-1
$$

with

$$
\sum_{i=1}^{N} \alpha_{i}=0
$$


With a procedure analogous to the one of section 5.1, we obtain the explicit formula

$$
\Xi_{N}\left(h_{2}, \cdots, h_{N}\right)=A_{N}\left(\hbar, \Lambda, h_{2}, \cdots h_{N-1}\right) \sum_{\mathbf{n} \in \mathcal{W}_{N} \cdot \gamma} \mathrm{e}^{J_{n}^{4 \mathrm{~d}}}
$$

The quantity $\mathrm{e}^{J_{\boldsymbol{n}}^{4 \mathrm{~d}}}$ is defined as follows. If $N$ is even we have:

$$
\mathrm{e}^{J_{\boldsymbol{n}}^{4 \mathrm{~d}}}=\exp \left(\frac{\mathrm{i}}{\hbar} \frac{\partial F_{\mathrm{NS}}}{\partial \boldsymbol{a}} \cdot \boldsymbol{n}\right) \prod_{\alpha \in \Delta_{+}}\left(2 \sinh \left(\frac{\pi \boldsymbol{a} \cdot \boldsymbol{\alpha}}{\hbar}\right)\right)^{-(\boldsymbol{n} \cdot \boldsymbol{\alpha})^{2}},
$$

while if $N$ is odd we have

$$
\mathrm{e}^{J_{\boldsymbol{n}}^{4 \mathrm{~d}}}=\exp \left(\frac{\mathrm{i}}{\hbar} \frac{\partial F_{\mathrm{NS}}}{\partial \boldsymbol{a}} \cdot \boldsymbol{n}-\frac{\pi}{\hbar} \boldsymbol{a} \cdot \boldsymbol{n}\right) \prod_{\alpha \in \Delta_{+}}\left(2 \sinh \left(\frac{\pi \boldsymbol{a} \cdot \boldsymbol{\alpha}}{\hbar}\right)\right)^{-(\boldsymbol{n} \cdot \boldsymbol{\alpha})^{2}}
$$

where $F_{\mathrm{NS}}$ is defined in (4.12). The quantity $A_{N}\left(\hbar, \Lambda, h_{2}, \cdots h_{N-1}\right)$ is an integration constant, analogous to $A(\hbar)$ in (5.6), which now depends on the moduli $h_{2}, \cdots, h_{N-1}$. The above spectral determinant vanishes precisely when the quantization conditions obtained in [18] are satisfied. When $N=2$ we recover exactly the result (5.6). When $N=3$ we have

$$
\begin{aligned}
\Xi_{3}\left(h_{3}, h_{2}\right)= & A_{3}\left(\hbar, \Lambda, h_{2}\right)\left[1+\frac{1-\mathrm{e}^{-2 \pi a_{1} / \hbar}}{1-\mathrm{e}^{-2 \pi\left(a_{1}+a_{2}\right) / \hbar}} \mathrm{e}^{-2 \pi a_{2} / \hbar} \mathrm{e}^{\mathrm{i} \phi_{2}}+\frac{1-\mathrm{e}^{-2 \pi a_{2} / \hbar}}{1-\mathrm{e}^{-2 \pi\left(a_{1}+a_{2}\right) / \hbar}} \mathrm{e}^{\mathrm{i} \phi_{1}}\right] \\
& \times \operatorname{csch}\left(\frac{\pi a_{1}}{\hbar}\right) \operatorname{csch}\left(\frac{\pi a_{2}}{\hbar}\right) \mathrm{e}^{-\frac{\pi\left(a_{1}-a_{2}\right)}{3 \hbar}-\mathrm{i} \frac{1}{3}\left(\phi_{1}+\phi_{2}\right)},
\end{aligned}
$$

where $\phi_{i}, i=1,2$, are defined as [18]

$$
\phi_{1}\left(a_{1}, a_{2} ; \hbar\right)=\frac{1}{\hbar}\left(\frac{\partial F_{\mathrm{NS}}}{\partial a_{2}}-2 \frac{\partial F_{\mathrm{NS}}}{\partial a_{1}}\right), \quad \phi_{2}\left(a_{1}, a_{2} ; \hbar\right)=\frac{1}{\hbar}\left(2 \frac{\partial F_{\mathrm{NS}}}{\partial a_{2}}-\frac{\partial F_{\mathrm{NS}}}{\partial a_{1}}\right) .
$$

We have tested (A.14) by expanding the r.h.s of (A.14) around $h_{3}=0$ and comparing with the numerical values of the spectral traces. We find perfect agreement.

\section{B Zamolodchikov's TBA equation for the modified Mathieu equation}

In [116] Zamolodchikov considered the thermodynamic TBA ansatz for the sinh-Gordon model. This model depends on the parameter $b \in \mathbb{C}$, and we introduce

$$
Q=b+\frac{1}{b},
$$

as well as

$$
p=\frac{b^{2}}{1+b^{2}}, \quad a=1-2 p=\frac{1-b^{2}}{1+b^{2}} .
$$

The TBA equation for this theory is given by

$$
\epsilon(\theta)=m R \cosh (\theta)-(\phi \star L)(\theta) .
$$

In this equation, $R$ is the radius of the circle where the theory lives, $m$ is the mass of the particle in the spectrum,

$$
L(\theta)=\log \left(1+\mathrm{e}^{-\epsilon(\theta)}\right)
$$


and

$$
\phi(\theta)=\frac{1}{2 \pi}\left(\frac{1}{\cosh (\theta-\mathrm{i} \pi a / 2)}+\frac{1}{\cosh (\theta+\mathrm{i} \pi a / 2)}\right)=\frac{1}{2 \pi} \frac{4 \sin (\pi p) \cosh (\theta)}{\cosh (2 \theta)-\cos (2 \pi p)} .
$$

The $\star$ in (B.3) denotes, as it is standard, the convolution

$$
(f \star g)(\theta)=\int_{\mathbb{R}} f\left(\theta-\theta^{\prime}\right) g\left(\theta^{\prime}\right) \mathrm{d} \theta^{\prime} .
$$

The ground state energy is then given by

$$
E(R)=-\frac{m}{2 \pi} \int_{\mathbb{R}} \cosh (\theta) L(\theta) \mathrm{d} \theta
$$

and the effective central charge is

$$
c_{\mathrm{eff}}=-\frac{6 R}{\pi} E(R)
$$

The formal conformal limit of the above TBA equation was analyzed in [19] in relation to the generalized Mathieu equation

$$
-u^{\prime \prime}(x)+\left(\mu_{-} \mathrm{e}^{-b x}+\mu_{+} \mathrm{e}^{b x}\right) u(x)=-P^{2} u(x) .
$$

The parameters $\mu_{ \pm}$have the following obvious symmetry

$$
\mu_{+} \rightarrow \mu_{+} \mathrm{e}^{-\varepsilon / b}, \quad \mu_{-} \rightarrow \mu_{-} \mathrm{e}^{+\varepsilon b}, \quad x \rightarrow x+\varepsilon,
$$

and therefore only the combination

$$
\mu=\mu_{+}^{b} \mu_{-}^{1 / b}
$$

matters. The parameter $b$ is identified with the parameter of the sinh-Gordon model, $\mu$ corresponds to its coupling constant, while the energy

$$
E=-P^{2}
$$

is identified with the Liouville momentum, and enters into the effective central charge of the theory, see (B.28). In the conformal limit, the TBA equation (B.3) becomes

$$
\epsilon(\theta)=\pi \mathrm{e}^{\theta}-2(\phi \star L)(\theta) .
$$

The dependence on $P$ comes through as the boundary condition of the TBA solution when $\theta \rightarrow-\infty$

$$
\epsilon(\theta) \sim 4 Q P \theta-2 C(P)+\cdots
$$

where $P>0$ and

$$
C(P)=\log \frac{\Gamma(2 P) \Gamma(1+2 P)}{2 \pi}+4 P \log \frac{16 \sqrt{\pi}}{\Gamma(1 / 4)^{2}} .
$$


It is then argued in [116] that the Fredholm determinant of the generalized Mathieu equation is given by [19]

$$
\Xi(\mu, P)=\frac{X(\mu, P)}{X(\mu, 0)},
$$

where

$$
X(\mu, P)=\exp [-\epsilon(\theta, P) / 2]
$$

and $\mu$ is related to $\theta$ by (5.30). We have indicated the explicit dependence of $\epsilon$ on $P$ through the boundary condition (B.14).

The ordinary modified Mathieu equation is obtained when

$$
b=1, \quad \mu_{-}=\mu_{+}=\mu .
$$

Let us focus on this case. The TBA equation becomes

$$
\epsilon(\theta)=\pi \mathrm{e}^{\theta}-\int_{\mathbb{R}} \frac{L\left(\theta^{\prime}\right)}{\cosh \left(\theta-\theta^{\prime}\right)} \frac{\mathrm{d} \theta^{\prime}}{\pi} .
$$

To impose the boundary condition (B.14), we use a trick due to Zamolodchikov. We first note that, as a consequence of (B.14), we have

$$
L(\theta) \sim-8 P \theta+2 C(P), \quad \theta \rightarrow-\infty,
$$

and we introduce the function

$$
L_{0}(\theta)=4 P \log \left(1+\mathrm{e}^{-2 \theta}\right)
$$

which has the same leading asymptotics than $L(\theta)$,

$$
L_{0}(\theta) \sim-8 P \theta+\mathcal{O}\left(\mathrm{e}^{-|\theta|}\right), \quad \theta \rightarrow-\infty .
$$

We have

$$
f_{0}=2 \phi \star L_{0}=8 P \log \left(1+\mathrm{e}^{-\theta}\right),
$$

and we can rewrite the TBA equation as

$$
\epsilon(\theta)=\pi \mathrm{e}^{\theta}-f_{0}-2 \phi \star\left(L-L_{0}\right) .
$$

This has by construction the right asymptotic behavior (B.14).

One property of (B.19) which is relevant for our analysis is the following. The asymptotic behavior of the solution $\epsilon(\theta)$ as $\theta \rightarrow \infty$ is of the form

$$
\epsilon(\theta)=\pi \mathrm{e}^{\theta}+\epsilon^{(1)} \mathrm{e}^{-\theta}+\cdots,
$$

where

$$
\epsilon^{(1)}=-\frac{2}{\pi} \int_{\mathbb{R}} \mathrm{e}^{\theta} \log \left(1+\mathrm{e}^{-\epsilon(\theta)}\right) \mathrm{d} \theta
$$


On the other hand, this correction is proportional to the effective central charge of the theory, ${ }^{10}$

$$
c_{\text {eff }}=\frac{6}{\pi} \int_{\mathbb{R}} \mathrm{e}^{\theta} \log \left(1+\mathrm{e}^{-\epsilon(\theta)}\right) \mathrm{d} \theta=-3 \epsilon^{(1)},
$$

which according to [19] can be computed in terms of $P$ only

$$
c_{\mathrm{eff}}=1+24 P^{2}
$$

This means that

$$
\epsilon^{(1)}=-\frac{1}{3}\left(1+24 P^{2}\right) .
$$

Open Access. This article is distributed under the terms of the Creative Commons Attribution License (CC-BY 4.0), which permits any use, distribution and reproduction in any medium, provided the original author(s) and source are credited.

\section{References}

[1] N.A. Nekrasov and S.L. Shatashvili, Quantization of Integrable Systems and Four Dimensional Gauge Theories, in 16th International Congress on Mathematical Physics, World Scientific, (2009), pp. 265-289, DOI [arXiv: 0908.4052] [INSPIRE].

[2] A. Grassi, Y. Hatsuda and M. Mariño, Topological Strings from Quantum Mechanics, Annales Henri Poincaré 17 (2016) 3177 [arXiv: 1410.3382] [INSPIRE].

[3] S. Codesido, A. Grassi and M. Mariño, Spectral Theory and Mirror Curves of Higher Genus, Annales Henri Poincaré 18 (2017) 559 [arXiv: 1507.02096] [INSPIRE].

[4] M. Mariño, Spectral Theory and Mirror Symmetry, Proc. Symp. Pure Math. 98 (2018) 259 [arXiv: 1506. 07757] [INSPIRE].

[5] D. Gaiotto, G.W. Moore and A. Neitzke, Four-dimensional wall-crossing via three-dimensional field theory, Commun. Math. Phys. 299 (2010) 163 [arXiv:0807.4723] [INSPIRE].

[6] D. Gaiotto, G.W. Moore and A. Neitzke, Wall-crossing, Hitchin Systems and the WKB Approximation, arXiv:0907.3987 [INSPIRE].

[7] D. Gaiotto, Opers and TBA, arXiv:1403.6137 [INSPIRE].

[8] K. Ito, M. Mariño and H. Shu, TBA equations and resurgent Quantum Mechanics, JHEP 01 (2019) 228 [arXiv:1811.04812] [INSPIRE].

[9] M. Mariño, From resurgence to BPS states, talk given at the conference Strings 2019, Brussels, Belgium 2019 https://livestream.com/accounts/7777696/events/8742238/videos/193704304.

[10] M. Mariño and S. Zakany, Matrix models from operators and topological strings, Annales Henri Poincaré 17 (2016) 1075 [arXiv: 1502.02958] [INSPIRE].

[11] A. Mironov and A. Morozov, Nekrasov Functions and Exact Bohr-Zommerfeld Integrals, JHEP 04 (2010) 040 [arXiv:0910.5670] [InSPIRE].

\footnotetext{
${ }^{10}$ There is a factor of 2 missing in eq. (4.4) of [19].
} 
[12] W. He and Y.-G. Miao, Mathieu equation and Elliptic curve, Commun. Theor. Phys. 58 (2012) 827 [arXiv : 1006.5185] [inSPIRE].

[13] M.-x. Huang, On Gauge Theory and Topological String in Nekrasov-Shatashvili Limit, JHEP 06 (2012) 152 [arXiv:1205.3652] [INSPIRE].

[14] G. Başar and G.V. Dunne, Resurgence and the Nekrasov-Shatashvili limit: connecting weak and strong coupling in the Mathieu and Lamé systems, JHEP 02 (2015) 160 [arXiv: 1501.05671] [INSPIRE].

[15] A.-K. Kashani-Poor and J. Troost, Pure $\mathcal{N}=2$ super Yang-Mills and exact WKB, JHEP 08 (2015) 160 [arXiv: 1504.08324] [INSPIRE].

[16] S.K. Ashok, D.P. Jatkar, R.R. John, M. Raman and J. Troost, Exact WKB analysis of $\mathcal{N}=2$ gauge theories, JHEP 07 (2016) 115 [arXiv: 1604.05520] [INSPIRE].

[17] S. Codesido, M. Mariño and R. Schiappa, Non-Perturbative Quantum Mechanics from Non-Perturbative Strings, Annales Henri Poincaré 20 (2019) 543 [arXiv:1712.02603] [INSPIRE].

[18] A. Grassi and M. Mariño, A Solvable Deformation of Quantum Mechanics, SIGMA 15 (2019) 025 [arXiv: 1806.01407] [inSPIRE].

[19] A.B. Zamolodchikov, Generalized Mathieu equations and Liouville TBA, in Quantum Field Theories in Two Dimensions, vol. 2. World Scientific, (2012).

[20] A. Voros, Spectre de l'équation de Schrödinger et méthode BKW, Publications Mathématiques d'Orsay, (1981).

[21] A. Voros, The return of the quartic oscillator. The complex WKB method, Annales de l'I.H.P. Physique Théorique 39 (1983) 211.

[22] E. Delabaere, H. Dillinger and F. Pham, Exact semiclassical expansions for one-dimensional quantum oscillators, J. Math. Phys. 38 (1997) 6126.

[23] K. Iwaki and T. Nakanishi, Exact WKB analysis and cluster algebras, J. Phys. A 47 (2014) 474009.

[24] R. Balian, G. Parisi and A. Voros, Quartic oscillator, in Feynman Path Integrals, vol. 106, Springer-Verlag, (1979), pp. 337-360.

[25] W. Lerche, Introduction to Seiberg-Witten theory and its stringy origin, Nucl. Phys. B Proc. Suppl. 55 (1997) 83 [hep-th/9611190] [INSPIRE].

[26] A. Marshakov, Seiberg-Witten theory and integrable systems, World Scientific, (1999).

[27] N. Seiberg and E. Witten, Electric-magnetic duality, monopole condensation and confinement in $N=2$ supersymmetric Yang-Mills theory, Nucl. Phys. B 426 (1994) 19 [Erratum ibid. 430 (1994) 485] [hep-th/9407087] [INSPIRE].

[28] M. Matone, Instantons and recursion relations in $N=2$ SUSY gauge theory, Phys. Lett. B 357 (1995) 342 [hep-th/9506102] [INSPIRE].

[29] R. Flume, F. Fucito, J.F. Morales and R. Poghossian, Matone's relation in the presence of gravitational couplings, JHEP 04 (2004) 008 [hep-th/0403057] [INSPIRE].

[30] G. Başar, G.V. Dunne and M. Ünsal, Quantum Geometry of Resurgent Perturbative/Nonperturbative Relations, JHEP 05 (2017) 087 [arXiv:1701.06572] [INSPIRE]. 
[31] A. Gorsky and A. Milekhin, RG-Whitham dynamics and complex Hamiltonian systems, Nucl. Phys. B 895 (2015) 33 [arXiv:1408.0425] [INSPIRE].

[32] S. Codesido and M. Mariño, Holomorphic Anomaly and Quantum Mechanics, J. Phys. A 51 (2018) 055402 [arXiv: 1612.07687] [INSPIRE].

[33] I. Aniceto, G. Başar and R. Schiappa, A Primer on Resurgent Transseries and Their Asymptotics, Phys. Rept. 809 (2019) 1 [arXiv:1802.10441] [INSPIRE].

[34] H.-Y. Chen, N. Dorey and K. Petunin, Wall Crossing and Instantons in Compactified Gauge Theory, JHEP 06 (2010) 024 [arXiv: 1004.0703] [inSPIRE].

[35] M. Kontsevich and Y. Soibelman, Stability structures, motivic Donaldson-Thomas invariants and cluster transformations, arXiv:0811.2435 [INSPIRE].

[36] F. Ferrari and A. Bilal, The strong coupling spectrum of the Seiberg-Witten theory, Nucl. Phys. B 469 (1996) 387 [hep-th/9602082] [INSPIRE].

[37] A. Klemm, W. Lerche, P. Mayr, C. Vafa and N.P. Warner, Selfdual strings and $N=2$ supersymmetric field theory, Nucl. Phys. B 477 (1996) 746 [hep-th/9604034] [inSPIRE].

[38] L.F. Alday, J. Maldacena, A. Sever and P. Vieira, Y-system for Scattering Amplitudes, J. Phys. A 43 (2010) 485401 [arXiv: 1002.2459] [InSPIRE].

[39] T.R. Klassen and E. Melzer, Purely Elastic Scattering Theories and their Ultraviolet Limits, Nucl. Phys. B 338 (1990) 485 [INSPIRE].

[40] M. Mariño, R. Schiappa and M. Weiss, Nonperturbative Effects and the Large-Order Behavior of Matrix Models and Topological Strings, Commun. Num. Theor. Phys. 2 (2008) 349 [arXiv:0711.1954] [INSPIRE].

[41] K. Ito and H. Shu, Generalized ODE/IM correspondence and its application to N=2 gauge theories, poster presented at the conference String Math 2018, Japan, June 18-22, 2018.

[42] L. Hollands and A. Neitzke, Exact WKB and abelianization for the $T_{3}$ equation, arXiv: 1906.04271 [INSPIRE].

[43] A.B. Zamolodchikov, Resonance factorized scattering and roaming trajectories, J. Phys. A 39 (2006) 12847 [INSPIRE].

[44] A.B. Zamolodchikov, Thermodynamic Bethe Ansatz in Relativistic Models. Scaling Three State Potts and Lee-yang Models, Nucl. Phys. B 342 (1990) 695 [InSPIRE].

[45] N.A. Nekrasov, Seiberg-Witten prepotential from instanton counting, Adv. Theor. Math. Phys. 7 (2003) 831 [hep-th/0206161] [INSPIRE].

[46] N. Nekrasov and A. Okounkov, Seiberg-Witten theory and random partitions, Prog. Math. 244 (2006) 525 [hep-th/0306238] [INSPIRE].

[47] R. Flume and R. Poghossian, An algorithm for the microscopic evaluation of the coefficients of the Seiberg-Witten prepotential, Int. J. Mod. Phys. A 18 (2003) 2541 [hep-th/0208176] [INSPIRE].

[48] U. Bruzzo, F. Fucito, J.F. Morales and A. Tanzini, Multiinstanton calculus and equivariant cohomology, JHEP 05 (2003) 054 [hep-th/0211108] [INSPIRE].

[49] M. Aganagic, M.C.N. Cheng, R. Dijkgraaf, D. Krefl and C. Vafa, Quantum Geometry of Refined Topological Strings, JHEP 11 (2012) 019 [arXiv:1105.0630] [INSPIRE]. 
[50] A.S. Losev, A. Marshakov and N.A. Nekrasov, Small instantons, little strings and free fermions, hep-th/0302191 [INSPIRE].

[51] M. Bullimore and H.-C. Kim, The Superconformal Index of the (2,0) Theory with Defects, JHEP 05 (2015) 048 [arXiv:1412.3872] [INSPIRE].

[52] M. Bullimore, H.-C. Kim and P. Koroteev, Defects and Quantum Seiberg-Witten Geometry, JHEP 05 (2015) 095 [arXiv: 1412.6081] [INSPIRE].

[53] F. Fucito, J.F. Morales and R. Poghossian, Wilson loops and chiral correlators on squashed spheres, JHEP 11 (2015) 064 [arXiv:1507.05426] [INSPIRE].

[54] A. Sciarappa, Bethe/Gauge correspondence in odd dimension: modular double, non-perturbative corrections and open topological strings, JHEP 10 (2016) 014 [arXiv: 1606.01000] [INSPIRE].

[55] Y. Hatsuda and M. Mariño, Exact quantization conditions for the relativistic Toda lattice, JHEP 05 (2016) 133 [arXiv: 1511.02860] [INSPIRE].

[56] K.K. Kozlowski and J. Teschner, TBA for the Toda chain, arXiv:1006.2906 [InSPIRE].

[57] M.C. Gutzwiller, The Quantum Mechanical Toda Lattice, Annals Phys. 124 (1980) 347 [INSPIRE].

[58] B. Simon, Notes on infinite determinants of Hilbert space operators, Adv. Math. 24 (1977) 244.

[59] A. Laptev, L. Schimmer and L.A. Takhtajan, Weyl type asymptotics and bounds for the eigenvalues of functional-difference operators for mirror curves, arXiv:1510.00045 [INSPIRE].

[60] J. Kallen and M. Mariño, Instanton effects and quantum spectral curves, Annales Henri Poincaré 17 (2016) 1037 [arXiv: 1308.6485] [INSPIRE].

[61] M.-x. Huang and X.-f. Wang, Topological Strings and Quantum Spectral Problems, JHEP 09 (2014) 150 [arXiv: 1406.6178] [INSPIRE].

[62] S. Codesido, A. Grassi and M. Mariño, Exact results in $\mathcal{N}=8$ Chern-Simons-matter theories and quantum geometry, JHEP 07 (2015) 011 [arXiv: 1409.1799] [INSPIRE].

[63] S.L. Lukyanov, unpublished.

[64] S.H. Katz, A. Klemm and C. Vafa, Geometric engineering of quantum field theories, Nucl. Phys. B 497 (1997) 173 [hep-th/9609239] [INSPIRE].

[65] G. Bonelli, A. Grassi and A. Tanzini, Seiberg-Witten theory as a Fermi gas, Lett. Math. Phys. 107 (2017) 1 [arXiv: 1603.01174] [InSPIRE].

[66] A. Voros, THE ZETA FUNCTION OF THE QUARTIC OSCILLATOR, Nucl. Phys. B 165 (1980) 209 [INSPIRE].

[67] A. Gorsky, A. Milekhin and N. Sopenko, Bands and gaps in Nekrasov partition function, JHEP 01 (2018) 133 [arXiv:1712.02936] [INSPIRE].

[68] M. Beccaria, On the large $\Omega$-deformations in the Nekrasov-Shatashvili limit of $\mathcal{N}=2^{*}$ $S Y M, J H E P 07$ (2016) 055 [arXiv: 1605.00077] [INSPIRE].

[69] P. Dorey and R. Tateo, Anharmonic oscillators, the thermodynamic Bethe ansatz and nonlinear integral equations, J. Phys. A 32 (1999) L419 [hep-th/9812211] [INSPIRE]. 
[70] P. Dorey, C. Dunning and R. Tateo, The ODE/IM Correspondence, J. Phys. A 40 (2007) R205 [hep-th/0703066] [INSPIRE].

[71] S.L. Lukyanov, Critical values of the Yang-Yang functional in the quantum sine-Gordon model, Nucl. Phys. B 853 (2011) 475 [arXiv:1105.2836] [INSPIRE].

[72] V.Y. Novokshenov, Movable poles of the solutions of painleve's equation of the third kind and their relation with mathieu functions, Funct. Anal. Appl. 20 (1986) 113-123.

[73] A. Sciarappa, Exact relativistic Toda chain eigenfunctions from Separation of Variables and gauge theory, JHEP 10 (2017) 116 [arXiv:1706.05142] [INSPIRE].

[74] A. Grassi and M. Mariño, The complex side of the TS/ST correspondence, J. Phys. A $\mathbf{5 2}$ (2019) 055402 [arXiv: 1708.08642] [INSPIRE].

[75] A. Grassi and J. Gu, BPS relations from spectral problems and blowup equations, Lett. Math. Phys. 109 (2019) 1271 [arXiv:1609.05914] [INSPIRE].

[76] O. Gamayun, N. Iorgov and O. Lisovyy, How instanton combinatorics solves Painlevé VI, V and IIIs, J. Phys. A 46 (2013) 335203 [arXiv: 1302.1832] [InSPIRE].

[77] A. Its, O. Lisovyy and Y. Tykhyy, Connection problem for the sine-Gordon/Painlevé III tau function and irregular conformal blocks, Int. Math. Res. Not. 18 (2015) 8903, [arXiv:1403.1235] [INSPIRE].

[78] P. Gavrylenko and O. Lisovyy, Pure SU(2) gauge theory partition function and generalized Bessel kernel, Proc. Symp. Pure Math. 18 (2018) 181 [arXiv:1705.01869] [InSPIRE].

[79] H. Nakajima and K. Yoshioka, Instanton counting on blowup. 1., Invent. Math. 162 (2005) 313 [math/0306198] [INSPIRE].

[80] H. Nakajima and K. Yoshioka, Instanton counting on blowup. II. K-theoretic partition function, math/0505553 [INSPIRE].

[81] H. Nakajima and K. Yoshioka, Lectures on instanton counting, in CRM Workshop on Algebraic Structures and Moduli Spaces, (2003) math/0311058 [INSPIRE].

[82] K. Sun, X. Wang and M.-x. Huang, Exact Quantization Conditions, Toric Calabi-Yau and Nonperturbative Topological String, JHEP 01 (2017) 061 [arXiv:1606.07330] [INSPIRE].

[83] X. Wang, G. Zhang and M.-x. Huang, New Exact Quantization Condition for Toric Calabi-Yau Geometries, Phys. Rev. Lett. 115 (2015) 121601 [arXiv:1505. 05360] [INSPIRE].

[84] S. Franco, Y. Hatsuda and M. Mariño, Exact quantization conditions for cluster integrable systems, J. Stat. Mech. 1606 (2016) 063107 [arXiv:1512.03061] [INSPIRE].

[85] M.A. Bershtein and A.I. Shchechkin, Bilinear equations on Painlevé $\tau$ functions from CFT, Commun. Math. Phys. 339 (2015) 1021 [arXiv: 1406.3008] [INSPIRE].

[86] M. Bershtein and A. Shchechkin, Painlevé equations from Nakajima-Yoshioka blowup relations, Lett. Math. Phys. 109 (2019) 2359 [arXiv:1811.04050] [INSPIRE].

[87] N. Nekrasov, Some applications of defects in supersymmetric gauge theory, talk given at IHES, France, January 2019.

[88] N. Iorgov, O. Lisovyy and J. Teschner, Isomonodromic tau-functions from Liouville conformal blocks, Commun. Math. Phys. 336 (2015) 671 [arXiv:1401.6104] [INSPIRE].

[89] M.A. Bershtein and A.I. Shchechkin, q-deformed Painlevé $\tau$ function and q-deformed conformal blocks, J. Phys. A 50 (2017) 085202 [arXiv: 1608.02566] [INSPIRE]. 
[90] M. Jimbo, H. Nagoya and H. Sakai, CFT approach to the q-Painlevé VI equation, Journal of Integrable Systems 2 (2017).

[91] M.R. Douglas, B. Fiol and C. Romelsberger, The spectrum of BPS branes on a noncompact Calabi-Yau, JHEP 09 (2005) 057 [hep-th/0003263] [INSPIRE].

[92] R. Eager, S.A. Selmani and J. Walcher, Exponential Networks and Representations of Quivers, JHEP 08 (2017) 063 [arXiv: 1611.06177] [INSPIRE].

[93] S. Banerjee, P. Longhi and M. Romo, Exploring 5d BPS Spectra with Exponential Networks, Annales Henri Poincaré 20 (2019) 4055 [arXiv: 1811.02875] [INSPIRE].

[94] T. Dimofte, S. Gukov, J. Lenells and D. Zagier, Exact Results for Perturbative Chern-Simons Theory with Complex Gauge Group, Commun. Num. Theor. Phys. 3 (2009) 363 [arXiv: 0903.2472] [INSPIRE].

[95] J. Ellegaard Andersen and R. Kashaev, A TQFT from Quantum Teichmüller Theory, Commun. Math. Phys. 330 (2014) 887 [arXiv:1109.6295] [INSPIRE].

[96] S. Gukov, M. Mariño and P. Putrov, Resurgence in complex Chern-Simons theory, arXiv: 1605.07615 [INSPIRE].

[97] D. Gang and Y. Hatsuda, S-duality resurgence in SL(2) Chern-Simons theory, JHEP 07 (2018) 053 [arXiv: 1710.09994] [INSPIRE].

[98] J.E. Andersen and W.E. Petersen, Resurgence Analysis of Quantum Invariants: Seifert Manifolds and Surgeries on The Figure Eight Knot, arXiv:1811.05376 [INSPIRE].

[99] T. Dimofte, D. Gaiotto and S. Gukov, Gauge Theories Labelled by Three-Manifolds, Commun. Math. Phys. 325 (2014) 367 [arXiv:1108.4389] [INSPIRE].

[100] A.B. Zamolodchikov, Painleve III and 2-D polymers, Nucl. Phys. B 432 (1994) 427 [hep-th/9409108] [INSPIRE].

[101] P. Fendley, Airy functions in the thermodynamic Bethe ansatz, Lett. Math. Phys. 49 (1999) 229 [hep-th/9906114] [INSPIRE].

[102] S. Cecotti, A. Neitzke and C. Vafa, Twistorial topological strings and a $\mathrm{tt}^{*}$ geometry for $\mathcal{N}=2$ theories in 4d, Adv. Theor. Math. Phys. 20 (2016) 193 [arXiv:1412.4793] [inSPIRE].

[103] A. Litvinov, S. Lukyanov, N. Nekrasov and A. Zamolodchikov, Classical Conformal Blocks and Painleve VI, JHEP 07 (2014) 144 [arXiv:1309.4700] [inSPIRE].

[104] M. Lencsés and F. Novaes, Classical Conformal Blocks and Accessory Parameters from Isomonodromic Deformations, JHEP 04 (2018) 096 [arXiv: 1709. 03476] [INSPIRE].

[105] G. Bonelli, F. Del Monte, P. Gavrylenko and A. Tanzini, $\mathcal{N}=2^{*}$ gauge theory, free fermions on the torus and Painlevé VI, Commun. Math. Phys. 377 (2020) 1381 [arXiv: 1901.10497] [INSPIRE].

[106] M. Bershtein, P. Gavrylenko and A. Grassi, in preparation.

[107] G. Bonelli, O. Lisovyy, K. Maruyoshi, A. Sciarappa and A. Tanzini, On painlevé/gauge theory correspondence, Lett. Math. Phys. 107 (2017) 2359.

[108] P.C. Argyres and M.R. Douglas, New phenomena in SU(3) supersymmetric gauge theory, Nucl. Phys. B 448 (1995) 93 [hep-th/9505062] [inSPIRE].

[109] P.C. Argyres, M. Plesser, N. Seiberg and E. Witten, New $N=2$ superconformal field theories in four-dimensions, Nucl. Phys. B 461 (1996) 71 [hep-th/9511154] [INSPIRE]. 
[110] A. Grassi and J. Gu, Argyres-Douglas theories, Painlevé II and quantum mechanics, JHEP 02 (2019) 060 [arXiv: 1803.02320] [INSPIRE].

[111] K. Ito and H. Shu, ODE/IM correspondence and the Argyres-Douglas theory, JHEP 08 (2017) 071 [arXiv:1707.03596] [INSPIRE].

[112] D. Masoero, Poles of Integrale Tritronquee and Anharmonic Oscillators. Asymptotic localization from WKB analysis, Nonlinearity 23 (2010) 2501 [arXiv:1002.1042] [INSPIRE].

[113] D. Masoero, Poles of integrale tritronquée and anharmonic oscillators. A WKB approach, J. Phys. A 43 (2010) 2501 [arXiv:0909.5537].

[114] V. Novokshenov, Poles of Tritronquée Solution to the Painlevé I Equation and Cubic Anharmonic Oscillator, Regul. Chaotic Dyn. 15 (2010) 390.

[115] C.M. Bender and J. Komijani, Painlevé Transcendents and PT-Symmetric Hamiltonians, J. Phys. A 48 (2015) 475202 [arXiv: 1502.04089] [InSPIRE].

[116] A.B. Zamolodchikov, On the thermodynamic Bethe ansatz equation in sinh-Gordon model, J. Phys. A 39 (2006) 12863 [hep-th/0005181] [INSPIRE]. 